\title{
THE SPACE OF ALL SELF-HOMEOMORPHISMS OF A TWO-CELL WHICH FIX THE CELL'S BOUNDARY IS AN ABSOLUTE RETRACT
}

\author{
BY \\ W. K. MASON( $\left.{ }^{1}\right)$
}

\begin{abstract}
The theorem mentioned in the title is proved. A corollary of the title theorem is: any homeomorphism between two compact subsets of the function space mentioned in the title can be extended to a homeomorphism of the function space onto itself.
\end{abstract}

1. Introduction. An interesting question in infinite-dimensional topology is the following: Is $H(D)$, the space of all homeomorphisms of a 2-disk $D$ onto itself which are the identity on the boundary of $D$, homeomorphic to $l_{2}$, the separable Hilbert space of square summable sequences [4, Question 22]; or much more generally, is $H(M)$, the space of homeomorphisms of a compact $n$-manifold onto itself (under the "sup norm" topology), locally homeomorphic to $l_{2}$ [22, Problem M1]? The answer to the last question is yes if $n=1$ [3], unknown if $n>1$. There are several partial results concerning these questions. It is well known that $H(M)$ is a complete separable metric space. The author [16] has shown that if $K$ is a sigmacompact subset of $H(M)$, then $H(M)-K$ is homeomorphic to $H(M)$. Geoghegan [10] has shown that $H(M) \times l_{2}$ is homeomorphic to $H(M)$. Edwards and Kirby [9] have shown that $H(M)$ is locally contractible. (Earlier, Dyer and Hamstrom [8] showed that $H(M)$ is locally contractible if $M$ is a 2-manifold with boundary. See also [11], [12].)

It is unknown whether or not $H(M)$ is an absolute neighborhood retract. In this paper we prove that $H(D)$ is an absolute retract (Theorem 4).

The basic idea of the proof is to show that for every open covering $\alpha$ of $H(D)$ there is a locally finite polyhedron $\alpha$-dominating $H(D)$. (See §2.) We make strong use of a selection theorem of Michael (Theorem 6 of this paper) in the main proof of this paper which is contained in $\$ 4$. The verification that Michael's theorem may be applied is postponed until §5. Possibly readers will supply their own verifications and will not have to read $\$ 5$. $\$ 6$ concludes the paper with a corollary.

Received by the editors May 18, 1970.

AMS 1970 subject classifications. Primary 57A05, 58D10.

Key words and phrases. Retract, absolute retract, two-cell, two-manifold, two-manifold function space, space of homeomorphisms, infinite-dimensional manifold.

(1) Research partially supported by NSF GP 11699. 
All homeomorphism spaces mentioned in this paper will be topologized by the "sup norm" distance function (see $\$ 2$ ). We shall use without explicit mention elementary results concerning arcwise accessibility and separation of sets in the plane (see Chapters 5-6 of [18], or Chapter 6 of [21]).

2. Definitions and notation. The statement that a metric space $X$ is an absolute retract (AR) means that whenever $X$ is embedded as a closed subset $Z_{0}$ of a metric space $Z$, there is a retraction of $Z$ onto $Z_{0}$. The statement that a metric space $X$ is an absolute neighborhood retract (ANR) means that whenever $X$ is embedded as a closed subset $Z_{0}$ of a metric space $Z$, there is a retraction of an open neighborhood of $Z_{0}$ onto $Z_{0}$.

$I$ or $[0,1]$ denotes the closed unit interval, and $R^{2}$ the plane (Euclidean 2-space). If $f_{1}, f_{2}: A \rightarrow B$ are homeomorphisms of the compact metric space $A$ into the metric space $B$, then dist $\left(f_{1}, f_{2}\right)$, the distance between $f_{1}$ and $f_{2}$, will be $\sup _{x \in A}$ dist $\left(f_{1}(x), f_{2}(x)\right)$. If $A$ and $B$ are complete metric spaces, with metrics $d_{1}, d_{2}$ resp., then a complete metric $d_{3}$ on $A \times B$ is given by

$$
d_{3}\left(\left(a_{1}, b_{1}\right),\left(a_{2}, b_{2}\right)\right)=d_{1}\left(a_{1}, a_{2}\right)+d_{2}\left(b_{1}, b_{2}\right), \quad a_{1}, a_{2} \in A, b_{1}, b_{2} \in B .
$$

Suppose $A$ and $B$ are point sets. Then $A+B$ denotes the union (sum) of $A$ and $B$; $\mathrm{Bd}(A)$ denotes the set of boundary points of $A$; Int $(A)$ denotes the set of interior points of $A$.

Suppose $C$ is a collection of subsets of a set $X$. Then $|C|$ denotes the set of all points $p$ of $X$ such that $p$ is in some element of $C$; if $\alpha \in C$, then st $(\alpha, C)$, the star of $\alpha$ in $C$, denotes the collection of all $\gamma \in C$ such that $\alpha \cap \gamma \neq \varnothing$.

A cover of a space will always mean an open cover; a cover $\gamma$ is a refinement of a cover $\alpha$ if every element of $\gamma$ is contained in some element of $\alpha$.

If $\gamma$ is a locally finite cover of a space $X$, then the nerve of $\gamma$ is the simplicial complex whose set of vertices is $\gamma$, and where elements $U_{1}, \ldots, U_{n}$ of $\gamma$ are vertices of a (closed) simplex $\sigma$ of the nerve if and only if $U_{1} \cap \cdots \cap U_{n} \neq \varnothing$. It is well known [7, Theorem 1.1] that there exists a standard barycentric map $b$ from $X$ into the nerve of $\gamma$ such that if $b(x) \in(\sigma-\mathrm{Bd}(\sigma))$ for some simplex $\sigma$ of the nerve, with vertices $U_{1}, \ldots, U_{n}$, then $x \in U_{1} \cap \cdots \cap U_{n}$.

By map we mean continuous function; an embedding of $A$ into $B$ is a homeomorphism from $A$ into $B$. An isotopy $\lambda$ of a space $A$ into (onto) a space $B$ is a map $\lambda: A \times I \rightarrow B$ such that for each $t \in I$, the map $\lambda_{t}$, defined by $\lambda_{t}(a)=\lambda(a, t)$, all $a \in A$, is a homeomorphism of $A$ into (onto) $B$.

Suppose $X$ is a space and $\alpha$ is a cover of $X$. Then a map $f: X \rightarrow X$ is limited by $\alpha$ if for each $x \in X$ there is an element $U$ of $\alpha$ such that $x$ and $f(x)$ are points of $U$. A homotopy $\lambda: X \times I \rightarrow X$ is an $\alpha$-homotopy if for each $x \in X$ there is an element $U$ of $\alpha$ such that $\lambda(x, t) \in U$ for all $t \in I$. A space $Y \alpha$-dominates $X$ if there are maps $f: X \rightarrow Y$ and $g: Y \rightarrow X$, and an $\alpha$-homotopy $\lambda: X \times I \rightarrow X$ such that, for each $x \in X, \lambda(x, 0)=x$ and $\lambda(x, 1)=g f(x)$. 
A space $X$ is locally $n$-connected $\left(\mathrm{LC}^{n}\right)$ if for every $x \in X$ and every open set $W$ containing $x$, there is an open set $U$ containing $x$ such that every map of a $k$ sphere, $k \leqq n$, into $U$ is homotopic in $W$ to a constant map.

If $X$ is a space, the cone over $X$, denoted by $C(X)$, is the identification space formed from $X \times I$ by identifying $X \times\{1\}$ to a point.

An $\operatorname{arc} B$ is a spanning $\operatorname{arc}$ of a disk $Z$ if $B$ is contained in $Z$, the endpoints of $B$ are contained in $\mathrm{Bd}(Z)$, and except for its endpoints $B$ misses $\mathrm{Bd}(Z)$.

3. $H(D)$ is an absolute retract. Following Alexander [1] let us define the Alexander map $A L: H(D) \times H(D) \times I \rightarrow H(D)$ by $A L(f, g, 0)(x)=f(x)$, all $(f, g)$ $\in H(D) \times H(D), \quad x \in D ;$ and $A L(f, g, t)(x)=f\left(t \cdot\left[f^{-1} g(x / t)\right]\right), \quad 0<t \leqq 1, \quad x \in D$, $(f, g) \in H(D) \times H(D)$. Here we think of points $x \in R^{2}$ as being vectors based at the origin, $D$ as being the set $\left\{x \in R^{2}\right.$ : length $\left.x \leqq 1\right\}$, and we think of maps $f \in H(D)$ as being extended to $R^{2}-D$ by the identity.

The following lemma is easily proved.

LEMMA 1. Let $\alpha$ be a cover of $H(D)$. Then there is a refinement $\gamma$ of $\alpha$ such that if $\psi: H(D) \rightarrow H(D)$ is a map which is limited by $\gamma$ then the homotopy $\theta: H(D) \times I \rightarrow H(D)$ defined by $\theta(f, t)=A L(\psi(f), f, t)$ is an $\alpha$-homotopy between $\psi$ and the identity map.

(Proof omitted.)

In $\$ 4$ we shall define a basis HVT (Horizontal and Vertical Tube cover) for $H(D)$. In the present section we shall assume Theorems 2 and 3 concerning HVT. Theorems 2 and 3 will be proved in $\$ 4$.

THEOREM 2. Let $O_{1}, \ldots, O_{n}$ be a finite set of elements of the basis HVT. Then $\bigcap_{i=1}^{n} O_{i}$ is an element of $H V T$.

THEOREM 3. Let $U$ be an element of $H V T$ and $K$ a finite-dimensional compact subset of $U$. Then there is an embedding $\psi$ of the cone over $K$ into $U$ such that $\psi(f, 0)$ $=f$, for all $f \in K$.

THEOREM 4. $H(D)$ is an $A R$.

Proof. The homotopy $\theta: H(D) \times I \rightarrow H(D)$ defined by $\theta(f, t)=A L(f, \mathrm{Id}, t)$, for all $(f, t) \in H(D) \times I$, contracts $H(D)$ to a point. Therefore, since $H(D)$ is contractible, it suffices, by [6], to prove that $H(D)$ is an ANR. By a theorem of Hanner [13, Theorem 7.2] $H(D)$ is an ANR if for every cover $\alpha$ of $H(D)$ there is a locally finite polyhedron $\alpha$-dominating $H(D)$. (See $\$ 2$ for definitions.) By Lemma 1, it suffices to show that for every cover $\alpha$ of $H(D)$ there is a locally finite polyhedron $P$ and maps $b: H(D) \rightarrow P$ and $\psi: P \rightarrow H(D)$ such that $\psi b$ is limited by $\alpha$. 
Suppose then that $\alpha$ is a fixed cover of $H(D)$. We may assume that each element of $\alpha$ is also an element of the basis HVT. By [20, p. 980] there is a countable refinement $\gamma$ of $\alpha$ such that (a) if $h \in \gamma$, then st $(h, \gamma)$, the star of $h$ in $\gamma$, is a finite set, and (b) if $h \in \gamma$ then there is a $V \in \alpha$ such that $\mid$ st $(h, \gamma) \mid$ is contained in $V$. Let $P$ be the nerve of $\gamma$ and let $b: H(D) \rightarrow P$ be the standard barycentric map. Order the elements of $\gamma$, and for each $h_{i} \in \gamma$, let $V_{i}$ be an element of $\alpha$ such that $\mid$ st $\left(h_{i}, \gamma\right) \mid \subset V_{i}$.

We shall define the map $\psi: P \rightarrow H(D)$ by induction on the skeletons of $P$. For each vertex $\left(h_{i}\right)$ of $P$, let $\psi^{0}\left(\left(h_{i}\right)\right)$ be any point of the set $h_{i} \subset H(D)$. Now assume $\psi^{0}$ has been extended to a map $\psi^{n}: P^{n} \rightarrow H(D)\left(P^{n}\right.$ denotes $n$-skeleton of $\left.P\right)$ such that for each (closed) simplex $\sigma^{n}=\left(h_{\lambda_{0}}, \ldots, h_{\lambda_{n}}\right)$ we have

(a) $\psi^{n}\left(\sigma^{n}\right)$ is finite dimensional, and

(b) $\psi^{n}\left(\sigma^{n}\right) \subset \bigcap\left\{V_{i} \in \alpha: h_{i} \in \bigcap_{j=0}^{n}\right.$ st $\left.\left(h_{\lambda_{j}}, \gamma\right)\right\}$.

Now let $\sigma^{n+1}$ be any closed $n+1$ simplex of $P^{n+1}$. Assume the vertices of $\sigma^{n+1}$ are $h_{0}, \ldots, h_{n+1}$. Let $U=\bigcap\left\{V_{i} \in \alpha: h_{i} \in \bigcap_{j=0}^{n+1}\right.$ st $\left.\left(h_{j}, \gamma\right)\right\}$. By Theorem $2, U$ is an element of HVT. Since the image under $\psi^{n}$ of each $n$-simplex in the boundary of $\sigma^{n+1}$ is a finite-dimensional compact set, the image under $\psi^{n}$ of the boundary of $\sigma^{n+1}$ is a finite-dimensional compact set [15, Theorem III2] which we denote by $K$. Clearly $K \subset U$. By Theorem 3 there is an embedding $\lambda: C(K) \rightarrow U$ such that $\lambda(f, 0)=f$ for all $f \in K$. The simplex $\sigma^{n+1}$ may be thought of as the cone over its boundary, and so for $(x, t) \in \sigma^{n+1}$, let $\psi^{n+1}(x, t)=\lambda\left(\psi^{n}(x), t\right)$.

Extending over each $\sigma^{n+1}$ of $P^{n+1}$ in this way gives $\psi^{n+1}: P^{n+1} \rightarrow H(D)$ and completes the induction.

Now suppose $f \in H(D)$, and $h_{0}, \ldots, h_{n}$ are the elements of $\gamma$ which contain $f$. Let $U^{\prime}=\bigcap\left\{V_{i} \in \alpha: h_{i} \in \bigcap_{j=0}^{n}\right.$ st $\left.\left(h_{j}, \gamma\right)\right\}$. It is easy to check that (1) $\bigcap_{j=0}^{n} h_{j} \subset U^{\prime}$, so $f \in U^{\prime}$, and (2) $\psi b(f) \in U^{\prime}$. Also, $U^{\prime}$ is contained in an element of $\alpha$. Hence $\psi b$ is limited by $\alpha$.

The proof of Theorem 4 is complete.

4. The basis HVT. In this section we describe the basis HVT and prove Theorems 2 and 3. First, however, we make some preliminary definitions.

It is convenient to think of the disk $D$ now as being a rectangle in $R^{2}$ with horizontal and vertical sides. Following [18, Chapter $\mathrm{V}, \S 1]$ a grating $P$ on $D$ is formed by drawing a finite number of spanning segments across $D$, parallel to its sides. These spanning segments will be called the crosscuts of $P$. The 2 -cells of $P$ are the closures of the rectangular domains into which the interior of $D$ is divided by the crosscuts of $P$. The mesh of $P$ is the maximum of the diameters of the 2-cells of $P$. If $E$ is a subset of $D$, the star of $E$ in $P$, st $(E, P)$, is the collection of all 2-cells of $P$ which intersect $E$.

Let $P_{1}, P_{2}, \ldots$ be a fixed sequence of gratings on $D$ such that (a) the mesh of $P_{i}$ approaches zero as $i$ approaches infinity, and (b) if $l$ is a crosscut of some $P_{i}$, then $l$ is a crosscut of $P_{j}$ for all $j \geqq i$. 


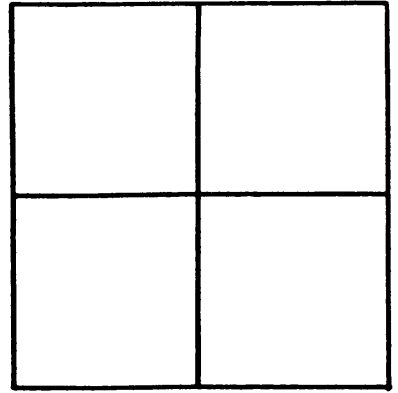

$P_{1}$

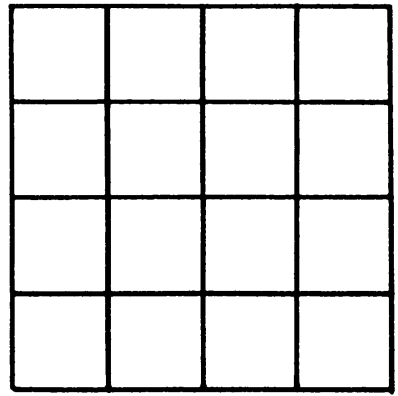

$P_{2}$

\section{Diagram 1}

For convenience of notation we shall also assume that, for each $i$, the set of crosscuts of $P_{i}$ is made up of an equal number of vertical and horizontal segments.

Let $\mathscr{H}$ be the collection of all polyhedral disks $H$ contained in $D$ such that $\mathrm{Bd}(H)$ is the union of a vertical segment in the left side of $\mathrm{Bd}(D)$, a vertical segment in the right side of $\mathrm{Bd}(D)$, and two polygonal spanning arcs of $D . \mathscr{H}$ is called the collection of "horizontal" tubes in $D$. Let $\mathscr{V}$ be the collection of all polyhedral disks $V$ contained in $D$ such that $\mathrm{Bd}(V)$ is the union of a horizontal segment in the top of $\mathrm{Bd}(D)$, a horizontal segment in the bottom of $\mathrm{Bd}(D)$, and two polygonal spanning arcs of $D . \mathscr{V}$ is called the collection of "vertical" tubes in $D$.

We now define the sets $O\left(P_{j} ; H_{1}, \ldots, H_{n} ; V_{1}, \ldots, V_{n}\right)$ which make up the basis HVT. Let $P_{j}$ be any grating in the sequence $P_{1}, P_{2}, \ldots$ Let $\left\{l_{1}, \ldots, l_{n}\right\}$ be the set of horizontal crosscuts of $P_{j}$, and $\left\{m_{1}, \ldots, m_{n}\right\}$ the set of vertical crosscuts of $P_{j}$. Let $\left\{H_{1}, \ldots, H_{n}\right\}$ be a set of $n$ elements of $\mathscr{H}$ such that $H_{i} \cap H_{j}=\varnothing, i \neq j$; and let $\left\{V_{1}, \ldots, V_{n}\right\}$ be a set of $n$ elements of $\mathscr{V}$ such that $V_{i} \cap V_{j}=\varnothing, i \neq j$. Then $O\left(P_{j} ; H_{1}, \ldots, H_{n} ; V_{1}, \ldots, V_{n}\right)$ is the set of all $f \in H(D)$ such that $f\left(l_{i}\right) \subset H_{i}$ - closure $\left(D-H_{i}\right), 1 \leqq i \leqq n$, and $f\left(m_{i}\right) \subset V_{i}$-closure $\left(D-V_{i}\right), 1 \leqq i \leqq n$. It is clear that $O\left(P_{j} ; H_{1}, \ldots, H_{n} ; V_{1}, \ldots, V_{n}\right)$ is an open subset of $H(D)$.

All sets of the form $O\left(P_{j} ; H_{1}, \ldots, H_{n} ; V_{1}, \ldots, V_{n}\right), P_{j} \in\left\{P_{1}, P_{2}, \ldots\right\},\left\{H_{1}, \ldots, H_{n}\right\}$ $\subset \mathscr{H},\left\{V_{1}, \ldots, V_{n}\right\} \subset \mathscr{V}$ make up the basis HVT.

LEMMA 5. HVT is a basis.

Proof. Suppose we are given $\varepsilon>0$ and $f \in H(D)$. We want to find an $O \in \mathrm{HVT}$ such that $f \in O$, and dist $(f, g)<\varepsilon$ for all $g \in O$.

Choose $\delta>0$ so that $\operatorname{dist}(f(x), f(y))<\varepsilon$ whenever dist $(x, y)<\delta$. Choose a grating $P_{j} \in\left\{P_{1}, P_{2}, \ldots\right\}$ such that if $C^{2}$ is any 2 -cell of $P_{j}$, then diam $\mid$ st $\left(C^{2}, P_{j}\right) \mid$ $<\delta$. Let $n$ denote the number of horizontal crosscuts of $P_{j}$. Choose "horizontal" tubes $H_{1}, \ldots, H_{n}$ of $\mathscr{H}$ such that (a) $H_{i} \cap H_{j}=\varnothing, i \neq j$, (b) $f\left(l_{i}\right) \subset H_{i}$ - closure $\left(D-H_{i}\right), 1 \leqq i \leqq n$ and (c) $f^{-1}\left(H_{i}\right) \subset \mid$ st $\left(l_{i}, P_{j}\right) \mid, 1 \leqq i \leqq n$. Choose "vertical" tubes $V_{1}, \ldots, V_{n}$ of $\mathscr{V}$ similarly. Then the set $O\left(P_{j} ; H_{1}, \ldots, H_{n} ; V_{1}, \ldots, V_{n}\right)$ is the required element of HVT. For, let $g \in O\left(P_{j} ; H_{1}, \ldots, H_{n} ; V_{1}, \ldots, V_{n}\right)$, and 
$x_{0} \in D$. Let $C^{2}$ be a 2-cell of $P_{j}$ containing $x_{0}$. It is easily seen that $f^{-1} g\left(\operatorname{Bd}\left(C^{2}\right)\right)$ $\subset \mid$ st $\left(C^{2}, P_{j}\right) \mid$, hence $f^{-1} g\left(C^{2}\right) \subset \mid$ st $\left(C^{2}, P_{j}\right) \mid$. Hence $\operatorname{dist}\left(f^{-1} g\left(x_{0}\right), x_{0}\right)<\delta$, so $\operatorname{dist}\left(g\left(x_{0}\right), f\left(x_{0}\right)\right)<\varepsilon$.

The proof of Lemma 5 is complete.

Proof of Theorem 2. Let $U=\bigcap_{i=1}^{n} O_{i}$. We want to show $U \in$ HVT. Assume $U \neq \varnothing$. To simplify notation assume that $P_{i}$ is the grating associated with $O_{i}$, $1 \leqq i \leqq n$. Then every crosscut of $P_{i}$ is a crosscut of $P_{n}, 1 \leqq i \leqq n$. Let $l_{1}$ be a horizontal crosscut of $P_{n}$. For each $i, 1 \leqq i \leqq n$, let $H_{1}^{i}$ be the "horizontal" tube associated with $l_{1}$ and $O_{i}$, provided there is one ( $l_{1}$ may not be a crosscut of some $P_{i}$ 's), otherwise let $H_{1}^{i}=D$. Since $U \neq \varnothing$ we may choose $f \in U$. Then $f\left(l_{1}\right)$ is contained in the closure of some component $H_{1}$ of $D-\sum_{i=1}^{n} \mathrm{Bd}\left(H_{1}^{i}\right)$. It is then clear that (a) if $g \in U$ then $g\left(l_{1}\right) \subset H_{1}-$ closure $\left(D-H_{1}\right)$ and (b) if $h \in H(D)$, and $h\left(l_{1}\right) \subset H_{1}$ - closure $\left(D-H_{1}\right)$, then $h\left(l_{1}\right) \subset H_{1}^{i}-$ closure $\left(D-H_{1}^{i}\right), 1 \leqq i \leqq n$. Finally, for each $i$, $1 \leqq i \leqq n, \mathrm{Bd}\left(H_{1}^{i}\right)$ is a polygonal simple closed curve containing the endpoints of $l_{1}$, hence by [18, p. 168], Bd $\left(H_{1}\right)$ is a (polygonal) simple closed curve. It follows that $H_{1} \in \mathscr{H}$.

Continuing in this way we define a "horizontal" tube $H_{j}$ for each horizontal crosscut $l_{j}$ of $P_{n}$ (assume there are $r$ such crosscuts), and a "vertical" tube $V_{j}$ for each vertical crosscut $m_{j}, 1 \leqq j \leqq r$, of $P_{n}$. The "horizontal" tubes $H_{j}$ are pairwise disjoint since each $H_{j}$ is contained in a tube $H_{j}^{n}$ associated with $\bar{U}_{n}$, and similarly for the "vertical" tubes $V_{j}$. Thus $U=U\left(P_{n} ; H_{1}, \ldots, H_{r} ; V_{1}, \ldots, V_{r}\right)$ is an element of HVT.

The proof of Theorem 2 is complete.

The proof of Theorem 3 makes use of a selection theorem of Michael [17]. The version we state here (Theorem 6) is the one given in [11, p. 32].

THEOREM 6. Suppose that $A$ and $B$ are metric spaces, $A$ complete and the (covering) dimension of $B$ does not exceed $n+1$, and that $Z$ is a closed subset of $B$. Suppose, further, that $R$ is an open mapping of $A$ onto $B$ such that the collection of inverses under $R$ is equi-LC $C^{n}$ (see below) and $r$ is a mapping of $Z$ into $A$ such that for $z \in Z$, $r(z) \in R^{-1}(z)$. Then there is a neighborhood $U$ of $Z$ such that $r$ may be extended to a mapping $r^{\prime}$ of $U$ into A such that for $z \in U, r^{\prime}(z) \in R^{-1}(z)$. If each inverse under $R$ has the property that all its homotopy groups vanish (of order $<n+1)$, then $U$ may be taken to be the entire space $B$.

The collection of inverses under $R$ is said to be equi- $L C^{n}$ provided that if $b \in B, a \in R^{-1}(b)$ and $F$ is a neighborhood of $a$, then there is a neighborhood $G$ of $a$ such that if $b^{\prime} \in B$ then every mapping of a $k$-sphere, $k \leqq n$, into $R^{-1}\left(b^{\prime}\right) \cap G$ is homotopic in $R^{-1}\left(b^{\prime}\right) \cap F$ to a constant map.

Proof of Theorem 3. (See statement in §3.) Let $P \in\left\{P_{1}, P_{2}, \ldots\right\}$ be the grating associated with $U$. Let $l_{1}, \ldots, l_{n}$ and $m_{1}, \ldots, m_{n}$ be the horizontal and vertical crosscuts, resp., of $P$. Let $H_{1}, \ldots, H_{n}$ and $V_{1}, \ldots, V_{n}$ be the "horizontal" and "vertical" tubes, resp., associated with $U$. 
Step A. There is a small square $N$ contained in a 2-cell of the grating $P$ and an isotopy $\theta_{1}: K \times I \rightarrow U$ such that (a) $N \cap \mathrm{Bd}(D)$ is a segment, (b) $N \cap \sum_{i=1}^{n}\left(H_{i}+V_{i}\right)$ is empty, (c) $\theta_{1}(f, 0)=f$ for all $f \in K$, (d) if $f \in K$ then $\theta_{1}(f, 1)|\operatorname{Bd}(N)=\operatorname{Id}| \operatorname{Bd}(N)$, and (e) if $f, g \in K, f \neq g$, then $\theta_{1}(f, 1)\left|N \neq \theta_{1}(g, 1)\right| N$. $\theta_{1}$ and $N$ are obtained as follows. Let $A L: H(D) \times H(D) \times I \rightarrow H(D)$ be the Alexander map of Lemma 1 . Let $\mu_{1}: H(D) \times I \rightarrow H(D)$ be defined by $\mu_{1}(f, t)=A L(\operatorname{Id}, f, 1-t)$ all $(f, t) \in H(D)$ $\times I$. By the compactness of $K$, there is a $t_{0} \in(0,1)$ such that if $f \in K$, and $0 \leqq t \leqq t_{0}$, then $\mu_{1}(f, t) \in U$. We may choose a square $N$, satisfying (a) and (b), small enough so that $\mu_{1}\left(f, t_{0}\right)|N=\operatorname{Id}| N$ for all $f \in H(D)$. We may also assume, by the compactness of $K$, that $N$ is small enough so that if $f \in \mu_{1}\left(K \times\left\{t_{0}\right\}\right), g \in H(D)$, and $f=g$ on $D-N$, then $g \in U$. Let $F: N \rightarrow D$ be a fixed homeomorphism of $N$ onto $D$. Let $\mu_{2}: \mu_{1}\left(K \times\left\{t_{0}\right\}\right) \times I \rightarrow U$ be the isotopy defined by

$$
\begin{array}{rlrl}
\mu_{2}(f, t)(x) & =f(x) & & \text { for } x \in D-N, \\
& =\left(F^{-1} \circ A L(\operatorname{Id}, f, t) \circ F\right)(x) & \text { for } x \in N .
\end{array}
$$

Then $\mu_{2}(f, 0)=f$ for all $f \in \mu_{1}\left(K \times\left\{t_{0}\right\}\right)$, since $f \mid N=\mathrm{Id}$; and if $f \neq g$, then $\mu_{2}(f, 1)$ $\neq \mu_{2}(g, 1)$ on $N$. Then, for $f \in K, t \in I$, let $\theta_{1}(f, t)=\mu_{1}(f, t)$ if $0 \leqq t \leqq t_{0}$, and $\theta_{1}(f, t)$ $=\mu_{2}\left(\mu_{1}\left(f, t_{0}\right),\left(t-t_{0}\right) /\left(1-t_{0}\right)\right)$ if $t_{0}<t \leqq 1$.

Let $K_{1}=\theta_{1}(K \times\{1\})$. Then $K_{1}$ has the property that if $\lambda: K_{1} \times I \rightarrow U$ is a homotopy, and $\lambda(f, t)|N=f| N$ for all $t \in I, f \in K_{1}$, then $\lambda$ is an isotopy.

Step B. Our goal now is to shrink $K_{1}$ so that all the homeomorphisms in the image of $K_{1}$ agree when restricted to the horizontal crosscut $l_{1}$.

Let the arcs making up the boundary of the "horizontal" tube $H_{1}$ be denoted by $L, R, T, B$, where $L$ is in the left side of $\mathrm{Bd}(D), R$ is in the right side, $T$ is the "top" of $H_{1}$, and $B$ is the "bottom". Since the set $\left|\left\{f\left(l_{1}\right): f \in K_{1}\right\}\right|$ is a compact subset of $D$ disjoint from $T+B$, we may adjust $T$ and $B$ slightly by "pushing them in", so that every component of $H_{1} \cap \sum_{i=1}^{n} \mathrm{Bd}\left(V_{i}\right)$ is a (polygonal) spanning arc of $H_{1}$. Since $\mathrm{Bd}\left(H_{1}\right)$ and $\sum_{i=1}^{n} \mathrm{Bd}\left(V_{i}\right)$ are polygonal we see that $H_{1} \cap \sum_{i=1}^{n} \mathrm{Bd}\left(V_{i}\right)$ has only a finite number of components. (The "pushing in" step is not absolutely necessary, but it simplifies the remaining arguments.) The components of $H_{1} \cap \sum_{i=1}^{n} \operatorname{Bd}\left(V_{i}\right)$ may be partitioned into two collections, denoted by SEP and NSEP. The elements of $S E P$ are arcs which separate $L$ and $R$ in $H_{1}$ (and thus have endpoints in both $T$ and $B$ ). The elements of NSEP are arcs which do not separate $L$ and $R$ in $H_{1}$ (and thus have either both endpoints in $T$ or both in $B$ ).

We want to get an isotopy $\theta_{2}: K_{1} \times I \rightarrow U$ such that (a) $\theta_{2}(f, 0)=f$, all $f \in K_{1}$, (b) $\theta_{2}(f, t)=f$ outside $f^{-1}\left(H_{1}\right)$, all $(f, t) \in K_{1} \times I$, and (c) $\theta_{2}(f, 1)\left(l_{1}\right) \cap|N S E P|=\varnothing$, all $f \in K_{1}$.

Let $B_{1}$ be an element of NSEP. We first push $f\left(l_{1}\right)$ off $B_{1}$ for each $f \in K_{1}$.

Let $M O N$ be a fixed subset of $R^{2}$ consisting of (a) three disks $D_{1}, D_{2}, D_{3}$, with disjoint interiors, such that $D_{1} \cap D_{2}$ and $D_{2} \cap D_{3}$ are arcs, and $D_{1} \cap D_{2} \cap D_{3}$ is two points $e_{1}, e_{2}$, plus (b) two arcs ("stickers") $M_{1}, M_{2}$ such that $M_{i} \cap\left(D_{1}+D_{2}+D_{3}\right)=\left\{e_{i}\right\}, i=1$, 2. (See Diagram 2.) 


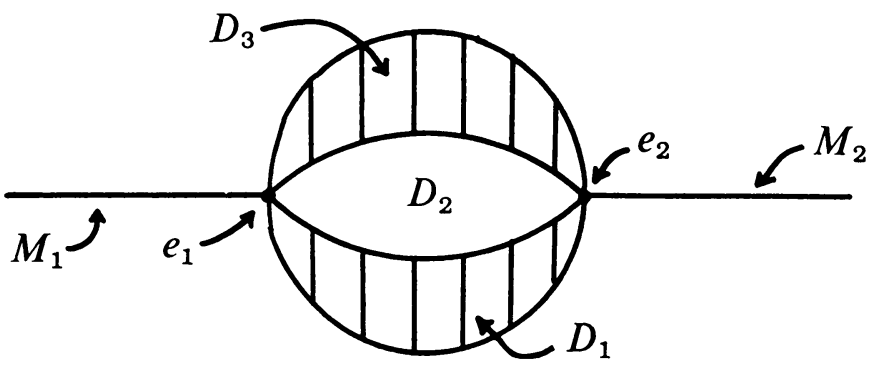

Diagram 2

Let $M_{3}$ be the arc $M_{1}+\left(D_{2} \cap D_{3}\right)+M_{2}$. Let $H\left(M O N, H_{1}\right)$ be the space of embeddings of $M O N$ into the tube $H_{1}$. Let $Q \subset K_{1} \times H\left(M O N, H_{1}\right)$ be the set of all $(f, j)$ in $K_{1} \times H\left(M O N, H_{1}\right)$ such that

(1) $j\left(M_{3}\right)=B_{1}, j$ (endpoint of $M_{3}$ in $M_{1}$ )=fixed endpoint $u$ of $B_{1}$,

(2) $j\left(D_{1}+D_{2}+D_{3}\right) \subset \operatorname{Int}\left(H_{1}\right)$,

(3) $j\left(D_{3}\right) \cap\left|N S E P-B_{1}\right|=\varnothing$,

(4) $f\left(\sum_{i=1}^{n} m_{i}\right) \cap j(M O N)=\varnothing$ (recall that the $m_{i}$ 's are the vertical crosscuts of $P$ ),

(5) $f\left(l_{1}\right) \cap j\left(D_{1}+M_{1}+M_{2}\right)=\varnothing$,

(6) $B_{1}$ separates $j\left(\operatorname{Int}\left(D_{1}\right)\right)$ and $L+R$ in $H_{1}$.

The set of all $(f, j)$ satisfying (2)-(5) is open, and the set satisfying (1) and (6) is closed, in $K_{1} \times H\left(M O N, H_{1}\right)$, hence $Q$ is a $G_{\delta}$ in a complete metric space, hence $Q$ is a complete metric space [14, Theorem 2-76].

Let $\pi_{1}: Q \rightarrow K_{1}$ be defined by $\pi_{1}(f, j)=f$, all $(f, j) \in Q$.

LEMMA 7. There is a mapping $r^{\prime}: K_{1} \rightarrow Q$ such that $\pi_{1} r^{\prime}=\mathrm{Id}: K_{1} \rightarrow K_{1}$.

Lemma 7 will be proved in $\S 5$. The proof consists of verifying that the hypotheses of Theorem 6 hold for the spaces $Q, K_{1}$, and the map $\pi_{1}$.

Let $\pi_{2}: Q \rightarrow H\left(M O N, H_{1}\right)$ be the map defined by $\pi_{2}(f, j)=j$, all $(f, j) \in Q$. Let $r: K_{1} \rightarrow H\left(M O N, H_{1}\right)$ be the map $r=\pi_{2} r^{\prime}$. For each $f \in K_{1}$, we shall use the embedding $r(f)$ to guide us in pushing $f\left(l_{1}\right)$ off $B_{1}$. Let $G: M O N \times I \rightarrow M O N$ be an isotopy of $M O N$ onto itself such that $G_{0}=\operatorname{Id}, G_{t}=\operatorname{Id}$ on $\operatorname{Bd}(M O N)$, all $t \in I$, and $G_{1}\left(D_{1}\right)=D_{1}+D_{2}$. Let $F: K_{1} \times I \rightarrow H(D)$ be the map defined by

$$
\begin{aligned}
F(f, t)(x) & =x & & \text { if } x \notin r(f)(M O N), \\
& =\left(r(f) \circ G_{t} \circ r(f)^{-1}\right)(x) & & \text { if } x \in r(f)(M O N)
\end{aligned}
$$

for all $(f, t) \in K_{1} \times I, x \in D$.

Finally, let $\theta_{2}: K_{1} \times I \rightarrow U$ be the isotopy defined by

$$
\theta_{2}(f, t)=F(f, t) \circ f, \quad \text { all }(f, t) \in K_{1} \times I .
$$

Then $\theta_{2}(f, t) \in U$, all $(f, t) \in K_{1} \times I$, because (a) $F(f, t)=\operatorname{Id}$ on $\sum_{i=1}^{n} f\left(m_{i}\right)$, since $r(f)(M O N) \cap \sum_{i=1}^{n} f\left(m_{i}\right)=\varnothing$ (property (4) of $Q$ ), and (b) $F(f, t)=$ Id outside $H_{1}$. Since $f\left(l_{1}\right) \cap r(f)\left(D_{1}+M_{1}+M_{2}\right)=\varnothing$, we see that $\theta_{2}(f, 1)\left(l_{1}\right) \cap r(f)\left(M_{3}\right)=$ 
$\theta_{2}(f, 1)\left(l_{1}\right) \cap B_{1}=\varnothing$. Also by property (3) of $Q, \theta_{2}(f, 1)\left(l_{1}\right)$ intersects no new elements of NSEP. By induction then we may assume that $\theta_{2}(f, 1)\left(l_{1}\right) \cap|N S E P|=\varnothing$, for all $f \in K_{1}$. Also, $\theta_{2}$ is an isotopy since $\theta_{2}(f, t)=f$ on the square $N$, all $(f, t) \in K_{1}$ $\times I$.

Let $K_{2}=\theta_{2}\left(K_{1} \times\{1\}\right)$.

Step C. Let $H_{1}^{\prime}$ be the closure of the component of $H_{1}-|N S E P|$ which has the vertical segments $L$ and $R$ in its boundary. Then

(a) $\mathrm{Bd}\left(H_{1}^{\prime}\right)$ is a polygonal simple closed curve [18, Theorem 11-8, p. 119],

(b) if $f \in K_{2}$, then $f\left(l_{1}\right)$ is a spanning arc of $H_{1}^{\prime}$ from $L$ to $R$, and

(c) the components of Int $\left(H_{1}^{\prime}\right) \cap \sum_{i=1}^{n} \mathrm{Bd}\left(V_{i}\right)$ are exactly the (open) arcs in SEP.

Let $A$ be a fixed spanning arc in $H_{1}^{\prime}$, with the same endpoints as $l_{1}$, such that $A$ intersects each arc in $S E P$ in exactly one point. We want to get a fixed embedding $j_{0}: l_{1} \rightarrow A$ and an isotopy $\theta_{3}: K_{2} \times I \rightarrow U$ such that $\theta_{3}(f, 1) \mid l_{1}=j_{0}$ for all $f \in K_{2}$.

For each $i, 1 \leqq i \leqq n$, let $p_{i}$ denote the point of intersection of $l_{1}$ and the vertical crosscut $m_{i}$. One may show that for each $i, 1 \leqq i \leqq n$, the set $\left\{f\left(p_{i}\right): f \in K_{2}\right\}$ is contained in just one component of $H_{1}^{\prime}-|S E P|$; denote this component by $S_{i}$. Let $j_{0}: l_{1} \rightarrow A$ be any fixed embedding such that $j_{0}\left(p_{i}\right) \in S_{i}, 1 \leqq i \leqq n$.

Let $H\left(A, H_{1}^{\prime}\right)$ denote the space of all embeddings of $A$ into $H_{1}^{\prime}$ which are fixed on the endpoints of $A$ and which take $A \cap \operatorname{Int}\left(H_{1}^{\prime}\right)$ into Int ( $\left.H_{1}^{\prime}\right)$. By Lemma 11(b) there is a homotopy $\lambda: K_{2} \times I \rightarrow H\left(A, H_{1}^{\prime}\right)$ such that for all $f \in K_{2}$, (a) $\lambda(f, 0)$ $=f \circ j_{0}^{-1}$, (b) $\lambda(f, 1)=\mathrm{Id}: A \rightarrow A$, and (c) if $A_{i}$ is the subarc of $A$ with endpoints $j_{0}\left(p_{i}\right)$ and $j_{0}\left(p_{i+1}\right)$, and $\alpha$ is any arc in $S E P$, then $\lambda(f, t)\left(A_{i}\right) \cap \alpha=\varnothing$ if and only if $A_{i} \cap \alpha=\varnothing$, all $t \in I$.

For each $f \in K_{2}, \lambda(f, t) \circ j_{0}$ will tell us where $f\left(l_{1}\right)$ is located during the isotopy $\theta_{3}$. We want to extend $\lambda(f, t)$ to an element of $H\left(H_{1}^{\prime}\right)$ for each $(f, t) \in K_{2} \times I$. (Here $H\left(H_{1}^{\prime}\right)$ denotes the space of all homeomorphisms of $D$ onto itself which are the identity outside $H_{1}^{\prime}$.) Because of certain technical difficulties we must obtain this extension in two stages.

Let $L E V$ be the set of all $(f, h)$ in $K_{2} \times H\left(H_{1}^{\prime}\right)$ such that

(1) $\lambda(f, 0)=h \mid A\left(=f \circ j_{0}^{-1}\right)$,

(2) $h^{-1}\left(f\left(\sum_{i=1}^{n} m_{i}\right)\right) \cap|S E P|=\varnothing$.

Let $\pi_{1}: L E V \rightarrow K_{2}, \pi_{2}: L E V \rightarrow H\left(H_{1}^{\prime}\right)$ be projection on the first and second coordinates resp.

LEMMA 8. There is a map $r_{1}^{\prime}: K_{2} \rightarrow L E V$ such that $\pi_{1} r_{1}^{\prime}=\mathrm{Id}: K_{2} \rightarrow K_{2}$.

The proof of Lemma 8 will be discussed in $\S 5$. Let $r_{1}=\pi_{2} r_{1}^{\prime}$. For each $f \in K_{2}$, let $\operatorname{MIS}(f)=r_{1}(f)^{-1}\left(f\left(\sum_{i=1}^{n} m_{i}\right)\right)$. Note that $\operatorname{MIS}(f) \cap|S E P|=\varnothing$.

Let $L I F$ be the set of all $(f, t, h) \in K_{2} \times I \times H\left(H_{1}^{\prime}\right)$ such that

(3) $\lambda(f, t)=h \mid A$,

(4) $h(\operatorname{MIS}(f)) \cap|S E P|=\varnothing$.

Let $\pi_{12}: L I F \rightarrow K_{2} \times I, \pi_{3}: L I F \rightarrow H\left(H_{1}^{\prime}\right)$ be projection on the first two and third 
coordinates resp. Let $\gamma: K_{2} \times\{0\} \rightarrow L I F$ be defined by $\gamma(f, 0)=\left(f, 0, r_{1}(f)\right)$, all $f \in K_{2}$.

LEMMA 9. There is a map $r_{2}^{\prime}: K_{2} \times I \rightarrow L I F$, which extends $\gamma$, such that $\pi_{12} r_{2}^{\prime}$ $=$ Id $: K_{2} \times I \rightarrow K_{2} \times I$.

Lemma 9 will be proved in $\S 5$. Let $r_{2}=\pi_{3} r_{2}^{\prime}$.

Finally, define the isotopy $\theta_{3}: K_{2} \times I \rightarrow U$ by $\theta_{3}(f, t)=r_{2}(f, t) \circ r_{1}(f)^{-1} \circ f$, all $(f, t) \in K_{2} \times I$.

$\theta_{3}$ is an isotopy since $\theta_{3}(f, t)=f$ on $N$, all $(f, t) \in K_{2} \times I$. Also, $\theta_{3}(f, 0)$ $=r_{1}(f) \circ r_{1}(f)^{-1} \circ f=f$, all $f \in K_{2} . \quad \theta_{3}(f, t) \in U$, for all $(f, t) \in K_{2} \times I$, since $\theta_{3}(f, t)\left(\sum_{i=1}^{n} m_{i}\right)$ misses $|S E P|$. Finally, $\theta_{3}(f, 1)\left|l_{1}=r_{2}(f, 1) \circ\left(j_{0} \circ f^{-1}\right) \circ f\right| l_{1}$ $=r_{2}(f, 1) \circ j_{0}=\lambda(f, 1) \circ j_{0}=j_{0}$, all $f \in K_{2}$.

Let $K_{3}=\theta_{3}\left(K_{2} \times\{1\}\right)$. Since $\theta_{3}(f, 1)=f$ outside $f^{-1}\left(H_{1}\right)$, we may assume by induction that if $f, g \in K_{3}$, then $g=f$ on all horizontal crosscuts $l_{i}, 1 \leqq i \leqq n$.

Step D. Our goal next is to shrink $K_{3}$ so that all homeomorphisms in the image of $K_{3}$ agree when restricted to any crosscut of the grating $P$.

Let $m_{1}^{\prime}$ be the subarc of the vertical crosscut $m_{1}$ such that the endpoints of $m_{1}^{\prime}$ are points of the horizontal crosscuts $l_{1}$ and $l_{2}$ (and $m_{1}^{\prime}$ interesects no other horizontal crosscuts). Thus, if $f \in K_{3}$, then $f\left(m_{1}^{\prime}\right)$ is in the "vertical" tube $V_{1}$, and $f\left(m_{1}^{\prime}\right)$ is a spanning arc of the disk $D_{1}^{\prime}$ bounded by $f\left(l_{1}\right)+f\left(l_{2}\right)$ plus two $\operatorname{arcs}$ in $\mathrm{Bd}(D)$. Let $D_{1}$ be the closure of the component of $D_{1}^{\prime}-\mathrm{Bd}\left(V_{1}\right)$ which has $f\left(m_{1}^{\prime}\right)$ as a spanning arc. By [18, Theorem 11.8, p. 119], $D_{1}$ is a disk. Note that if $g \in K_{3}$, then $g\left(m_{1}^{\prime}\right)$ is a spanning arc of $D_{1}$ with the same endpoints as $f\left(m_{1}^{\prime}\right)$, since $f=g$ on $l_{1}+l_{2}$.

Let $j_{1}: m_{1}^{\prime} \rightarrow D_{1}$ be a fixed embedding such that $j_{1}=f \mid m_{1}^{\prime}$ for some $f \in K_{3}$. We want to get an isotopy $\theta_{4}: K_{3} \times I \rightarrow U$ such that for all $(f, t) \in K_{3} \times I, \theta_{4}(f, t)=f$ outside $f^{-1}\left(D_{1}\right)$, and $\theta_{4}(f, 1) \mid m_{1}^{\prime}=j_{1}$.

Let $H\left(D_{1}\right)$ denote the space of all homeomorphisms of $D_{1}$ onto itself which are fixed on $\operatorname{Bd}\left(D_{1}\right)$. Let $V E R$ be the set of all $(f, h) \in K_{3} \times H\left(D_{1}\right)$ such that $h \mid A_{1}$ $=f \circ j^{-1}$, where $A_{1}=j_{1}\left(m_{1}^{\prime}\right)$. Let $\pi_{1}: V E R \rightarrow K_{3}$, and $\pi_{2}: V E R \rightarrow H\left(D_{1}\right)$ be the usual projections.

LEMMA 10. There is a map $r_{3}^{\prime}: K_{3} \rightarrow V E R$ such that $\pi_{1} r_{3}^{\prime}=\mathrm{Id}: K_{3} \rightarrow K_{3}$.

Lemma 10 will be proved in $\S 5$. Let $r_{3}: K_{3} \rightarrow H\left(D_{1}\right)$ be the map defined by $r_{3}=\pi_{2} r_{3}^{\prime}$. Let $A L^{\prime}: H\left(D_{1}\right) \times H\left(D_{1}\right) \times I \rightarrow H\left(D_{1}\right)$ be a map defined analogously to the Alexander map $A L$ of Lemma 1, except that we replace the disk $D$ in the definition of $A L$ by the disk $D_{1}$.

Define the isotopy $\theta_{4}: K_{3} \times I \rightarrow U$ by $\theta_{4}(f, t)=A L^{\prime}\left(\mathrm{Id}, r_{3}(f)^{-1}, t\right) \circ f$. (We extend $A L^{\prime}\left(\mathrm{Id}, r_{3}(f)^{-1}, t\right)$ outside $D_{1}$ by the identity.)

Then $\theta_{4}(f, t) \in U$ for all $(f, t) \in K_{3} \times I$, since $\theta_{4}(f, t)=f$ outside $f^{-1}\left(D_{1}\right)$. And $\theta_{4}(f, 1)\left|m_{1}^{\prime}=r_{3}(f)^{-1} \circ f\right| m_{1}^{\prime}=\left(j_{1} \circ f^{-1}\right) \circ f=j_{1} . \theta_{4}$ is an isotopy, since $\theta_{4}(f, t)=f$ on $N$ for all $(f, t) \in K_{3} \times I$.

Let $K_{4}=\theta_{4}\left(K_{3} \times\{1\}\right)$. Since $\theta_{4}(f, 1)=f$ outside $f^{-1}\left(D_{1}\right)$, all $f \in K_{3}$, we may assume 
by induction that if $f, g \in K_{4}$, then $f=g$ on all vertical crosscuts $m_{i}$ (and all horizontal crosscuts $\left.l_{i}\right), 1 \leqq i \leqq n$.

Step E. We shrink $K_{4}$ to a point in $U$. Let $g_{0}$ be a fixed element of $K_{4}$. Let $C_{1}^{2}, \ldots, C_{q}^{2}$ be the 2-cells of the grating $P$. For each $i, 1 \leqq i \leqq q$, let $F_{i}: C_{i}^{2} \rightarrow D$ be a fixed homeomorphism of $C_{i}^{2}$ onto $D$, and let $A L$ be the Alexander map of Lemma 1. Define a homotopy $\theta_{5}: K_{4} \times I \rightarrow U$ by

$$
\theta_{5}(f, t)(x)=g_{0} \circ F_{i}^{-1} \circ\left[A L\left(F_{i} g_{0}^{-1} f F_{i}^{-1}, \mathrm{Id}, t\right)\right] \circ F_{i}(x)
$$

for all $(f, t) \in K_{4} \times I, x \in C_{i}^{2}, 1 \leqq i \leqq q$.

Note that if $x \in \mathrm{Bd}\left(C_{i}^{2}\right), 1 \leqq i \leqq q$, and $f \in K_{4}$, then $g_{0}(x)=f(x)$, thus $\theta_{5}$ is well defined. Also, $\theta_{5}(f, 1)=g_{0}, \theta_{5}(f, 0)=f$, for all $f \in K_{4}$, and if $t \neq 1$, then $\theta_{5} \mid K_{4} \times\{t\}$ is a homeomorphism.

Finally, combining the homotopies $\theta_{1}, \ldots, \theta_{5}$ (an equation for this may be obtained as in $[4, \S 5])$ gives us a map $\psi_{1}: C(K) \rightarrow U$ such that $\psi_{1}(f, 0)=f$, all $f \in K$, and for each $t \in I, \psi_{1} \mid K \times\{t\}$ is a homeomorphism. But then, by Lemma 14 (see $\S 5)$, there is an embedding $\psi: C(K) \rightarrow U$ such that $\psi(f, 0)=f$ for all $f \in K$.

The proof of Theorem 3 is complete.

5. Some lemmas. In this section we prove the lemmas used in Theorem 3. First, however, we bring together in Lemmas 11 and 12 some ways of shrinking sets of homeomorphisms. The proofs of Lemmas 11 and 12 are based on various modifications of the Alexander map $A L$ of Lemma 1.

In Lemmas 11 and $12, Z$ will denote a fixed rectangle with vertical and horizontal sides, $Z_{1}$ a closed subset of $Z$ and $H\left(Z_{1}, Z\right)$ will be the space of all embeddings of $Z_{1}$ into $Z$ which are the identity on $Z_{1} \cap \mathrm{Bd}(Z)$, and which take $Z_{1} \cap \operatorname{Int}(Z)$ into Int $(Z)$. A subset $X$ of $H\left(Z_{1}, Z\right)$ has the compact shrinking property (C.S.P.) if given any compact subset $Y$ of $X$, there is a map $\lambda: C(Y) \rightarrow X$ such that $\lambda(f, 0)$ $=f$, all $f \in Y$, and $\lambda(f, 1)$ is the inclusion map of $Z_{1}$ into $Z$, all $f \in Y$.

LEMMA 11. Let $A$ be a horizontal spanning arc of $Z$, and $A_{1}, \ldots, A_{n}$ a subdivision of $A$ into subarcs with disjoint interiors. Let $V A N$ be a subset of $H\left(Z_{1}, Z\right)$. Then $V A N$ has the C.S.P. if one of (a), (b), (c) holds.

(a) $Z_{1}$ is the closure of a component of $Z-A$, and $V A N=H\left(Z_{1}, Z\right)$.

(b) There are vertical spanning segments $\gamma_{1}, \ldots, \gamma_{m}$ of $Z$ such that, for all $i, j$, $1 \leqq i \leqq n, 1 \leqq j \leqq m, \gamma_{j}$ misses the endpoints of $A_{i} . Z_{1}=A$, and

$$
\begin{aligned}
V A N=\left\{h \in H\left(Z_{1}, Z\right):\right. & h\left(A_{i}\right) \cap \gamma_{j}=\varnothing \\
& \text { if and only if } \left.A_{i} \cap \gamma_{j}=\varnothing, \text { all } i, j, 1 \leqq i \leqq n, 1 \leqq j \leqq m\right\} .
\end{aligned}
$$

(c) There are two collections $\left\{\alpha_{1}, \ldots, \alpha_{n-1}\right\}$ and $\left\{\gamma_{1}, \ldots, \gamma_{m}\right\}$ of pairwise disjoint spanning arcs of $Z$, each arc meeting the top and bottom of $\mathrm{Bd}(Z)$, such that for all $i, j, \gamma_{j} \cap \alpha_{i}=\varnothing$, and for all $i, 1 \leqq i \leqq n-1, \alpha_{i} \cap A$ is the right-hand endpoint of $A_{i}$. $Z_{1}=Z$ and $V A N=\left\{h \in H\left(Z_{1}, Z\right): h \mid A=\mathrm{Id}\right.$, and $h\left(\alpha_{i}\right) \cap \gamma_{j}=\varnothing$, all $\left.i, j\right\}$. 
Proof. Case (a). Identify $Z$ with the disk $D$ used in defining the Alexander map $A L$ of Lemma 1 . We may assume that if $x$ is a point (vector) of $Z_{1}$ then $x / t$ is either a point of $Z_{1}$ or a point outside $Z$, all $t \in(0,1]$. But then the map $\lambda(f, t)$ $=A L(\mathrm{Id}, f, 1-t)$ is well defined for all $(f, t) \in C(Y)$ and is the map required.

The proof for conditions (b) and (c) makes use of a canonical family of isotopies $R(\alpha, \beta, \gamma, t)$ of the plane onto itself. Let $\alpha, \beta, \gamma$ be vertical lines such that $\alpha$ is to the left of $\beta$ and $\beta$ is to the left of $\gamma$. Let $\{R(\alpha, \beta, \gamma, t)\}_{t \in[0,1]}$ be a continuous (in $t$ ) family of homeomorphisms of $R^{2}$ onto $R^{2}$ such that (1) if $l$ is any horizontal line in $R^{2}$, then $R(\alpha, \beta, \gamma, t)(l)=l$ for all $t \in I$, (2) if $v$ is any vertical line in $R^{2}$, then $R(\alpha, \beta, \gamma, t)(v)$ is a vertical line, all $t \in I,(3) R(\alpha, \beta, \gamma, 0)=\mathrm{Id},(4) R(\alpha, \beta, \gamma, t)(p)=p$ if $p$ is on or to the right of the vertical line $\gamma$, and (5) $R(\alpha, \beta, \gamma, 1)(\alpha)=\beta$.

Case (b). Assume inductively that we have a homotopy $\lambda_{n-2}: Y \times I \rightarrow V A N$ such that $\lambda_{n-2}(f, 0)=f$, all $f \in Y ; \lambda_{n-2}(f, 1)=$ Id on $A_{1}+\cdots+A_{n-2}$, all $f \in Y$; and if $x$ is a point of $A_{n-1}+A_{n}$, then $\lambda_{n-2}(f, 1)(x)$ is on or to the right of a vertical line $\omega_{n-2}$ through the right-hand endpoint of $A_{n-2}$, all $f \in Y$. Let $\omega_{n-1}$ be a vertical line through the right-hand endpoint of $A_{n-1}$. Let $\omega_{n-1}^{\prime}, \omega_{n-1}^{\prime \prime}$ be vertical lines to the left and right, resp., of $\omega_{n-1}$ such that none of the segments $\gamma_{j}, 1 \leqq j \leqq m$, intersects the (closed) strip between $\omega_{n-1}^{\prime}$ and $\omega_{n-1}^{\prime \prime}$.

For $t \in I$, let

$$
\begin{aligned}
Q_{t} & =R\left(\omega_{n-2}, \omega_{n-1}^{\prime}, \omega_{n-1}, t\right), & & \\
S_{t} & =R\left(\omega_{n-1}^{\prime}, \omega_{n-1}, \omega_{n-1}^{\prime \prime}, t\right), & & \\
\lambda_{n-1}^{\prime}(f, t) & =Q_{t} \circ \lambda_{n-2}(f, 1) \circ Q_{t}^{-1}, & & \text { all } f \in Y, \\
\lambda_{n-1}(f, t) & =\lambda_{n-2}(f, 3 t), & & 0 \leqq t \leqq \frac{1}{3}, \\
& =\lambda_{n-1}^{\prime}(f, 3 t-1), & & \frac{1}{3}<t \leqq \frac{2}{3}, \\
& =S_{(3 t-2)} \circ \lambda_{n-1}^{\prime}(f, 1) \circ S_{(3 t-2)}^{-1}, & & \frac{2}{3}<t \leqq 1
\end{aligned}
$$

(elements of $H\left(Z_{1}, Z\right)$ extend outside $Z$ by the identity).

As $t$ moves from $\frac{1}{3}$ to $\frac{2}{3}, \lambda_{n-1}(f, t)$ becomes the identity on the part of $A_{n-1}$ between $\omega_{n-2}$ and $\omega_{n-1}^{\prime}$, then as $t$ moves from $\frac{2}{3}$ to $1, \lambda_{n-1}(f, t)$ becomes the identity on all of $A_{n-1}$. Note that $Q_{t}$ moves no point of $A_{n}$, thus $\lambda_{n-1}(f, t) \in V A N, \frac{1}{3} \leqq t \leqq \frac{2}{3}$; and if $x \in A$ lies to the right of $\omega_{n-1}^{\prime}$, then $\lambda_{n-1}^{\prime}(f, 1)(x)$ lies to the right of $\omega_{n-1}^{\prime}$, thus $\lambda_{n-1}(f, t) \in V A N, \frac{2}{3}<t \leqq 1$. Thus $\lambda_{n-1}$ satisfies our inductive hypothesis.

Finally, we change $\lambda_{n-1}(f, 1)$ to the identity on $A_{n}$ (and thus on all of $A$ ), for each $f \in Y$. Identify the disk bounded by $\omega_{n-1}$, the right side of $\mathrm{Bd}(Z)$, and two arcs in the top and bottom of $\mathrm{Bd}(Z)$ with the disk $D$ used in defining the Alexander map $A L$ of Lemma 1 . We may assume that the $\operatorname{arc} A_{n}$ is a diameter of $D$. Then let $\lambda(f, t)=\lambda_{n-1}(f, 2 t), 0 \leqq t \leqq \frac{1}{2}$, and $\lambda(f, t)=A L\left(\mathrm{Id}, \lambda_{n-1}(f, 1), 2-2 t\right), \frac{1}{2}<t \leqq 1$, all $f \in Y . \lambda$ is the required map.

Case (c). Let $Y$ be a given compact subset of $V A N$. For each $i, 1 \leqq i \leqq n-1$, the compact set $\alpha_{i}+\left|\left\{f\left(\alpha_{i}\right): f \in Y\right\}\right|$ is disjoint from all arcs $\gamma_{j}, 1 \leqq j \leqq m$. Hence we may choose spanning arcs $\alpha_{i}^{\prime}, \alpha_{i}^{\prime \prime}$ of $Z$ separating $\alpha_{i}+\left|\left\{f\left(\alpha_{i}\right): f \in Y\right\}\right|$ from the left 
and right sides, resp., of $Z$ such that each of $\alpha_{i}^{\prime}, \alpha_{i}^{\prime \prime}$ intersects $A$ in one point, and such that the disk bounded by $\alpha_{i}^{\prime}+\alpha_{i}^{\prime \prime}$ plus two arcs of $\mathrm{Bd}(Z)$ intersects none of the arcs $\gamma_{j}, 1 \leqq j \leqq m$. We assume that the arcs $\alpha_{1}^{\prime}, \alpha_{1}^{\prime \prime}, \ldots, \alpha_{n-1}^{\prime \prime}$ are pairwise disjoint. We may also assume that the $\operatorname{arcs} \alpha_{i}^{\prime}, \alpha_{i}, \alpha_{i}^{\prime \prime}$ are vertical segments, for all $i, 1 \leqq i \leqq n-1$ (there is a homeomorphism of $Z$ onto itself taking the arcs $\alpha_{i}^{\prime}, \alpha_{i}, \alpha_{i}^{\prime \prime}$ onto vertical segments, for all $i, 1 \leqq i \leqq n-1$, while keeping the arc $A$ horizontal). Then for each $i$, $1 \leqq i \leqq n-1$, let $\omega_{i}^{\prime}, \omega_{i}, \omega_{i}^{\prime \prime}$ be vertical lines extending $\alpha_{i}^{\prime}, \alpha_{i}, \alpha_{i}^{\prime \prime}$ resp. Assume inductively that we have a homotopy $\lambda_{n-2}: Y \times I \rightarrow V A N$ such that $\lambda_{n-2}(f, 0)=f$, all $f \in Y$, and $\lambda_{n-2}(f, 1)(p)=p$ if $p$ lies to the left of the vertical line $\omega_{n-2}$, all $f \in Y$. Define $\lambda_{n-1}^{\prime}, \lambda_{n-1}$ exactly as in Case (b). Complete the argument as in Case (b).

The proof of Lemma 11 is complete.

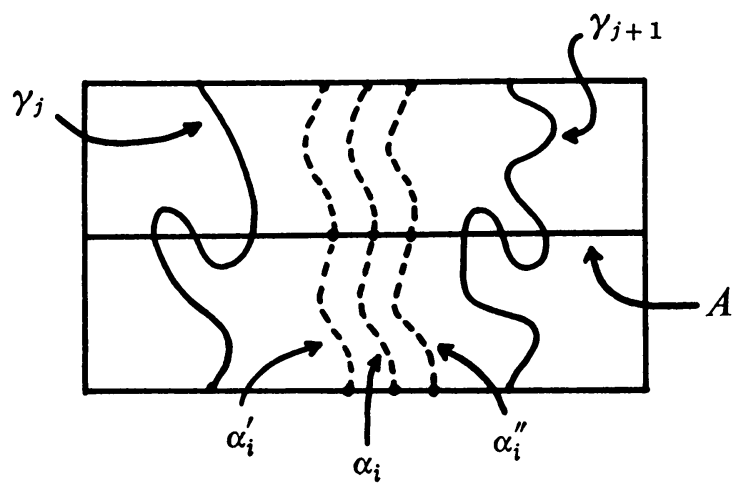

Diagram 3

LEMMA 12. (For definitions of $Z, Z_{1}, H\left(Z_{1}, Z\right)$, see beginning of $\S 5$.) Suppose $Z_{2}$ is a disk contained in $Z_{1}$ such that $Z_{2} \cap \mathrm{Bd}(Z)$ is an arc, and every point of $\operatorname{Bd}\left(Z_{2}\right)-\left(Z_{2} \cap \mathrm{Bd}(Z)\right)$ is an interior point of $Z_{1}$. Suppose $Z_{3}$ is a closed subset of $Z_{1}$ such that $Z_{2} \cap Z_{3}$ is at most the endpoints of $Z_{2} \cap \operatorname{Bd}(Z)$. Suppose $V A N$ is a subset of $H\left(Z_{1}, Z\right)$ satisfying either $(\mathrm{a})$ or $(\mathrm{b})$ below. Then if $Y$ is any compact subset of $V A N$, there is a homotopy $\lambda: Y \times I \rightarrow V A N$ such that for all $f \in Y, \lambda(f, 0)=f$, and $\lambda(f, 1) \mid Z_{2}=$ Id.

(a) $V A N=\left\{f \in H\left(Z_{1}, Z\right): f\left(Z_{2}\right) \cap Z_{3}=Z_{2} \cap Z_{3}\right\}$,

(b) $V A N=\left\{f \in H\left(Z_{1}, Z\right): Z_{2} \cap f\left(Z_{3}\right)=Z_{2} \cap Z_{3}\right\}$.

In addition, $\lambda$ may be chosen so that if $N$ is a preassigned neighborhood (in $Z$ ) of $Z_{2}$, then for all $x \in Z_{1}-N$, either $\lambda(f, t)(x)=f(x)$ or $\lambda(f, t)(x) \in N$, for all $(f, t) \in Y$ $\times I$.

Proof. "Push out" slightly on the disk $Z_{2}$ to obtain a new disk $Z_{2}^{\prime}$ in $Z_{1}$ such that $\operatorname{Bd}\left(Z_{2}^{\prime}\right) \cap \operatorname{Bd}\left(Z_{2}\right)=Z_{2} \cap \operatorname{Bd}(Z)=Z_{2}^{\prime} \cap \operatorname{Bd}(Z)$. (Thus $\operatorname{Bd}\left(Z_{2}^{\prime}\right)+\operatorname{Bd}(Z)$ is a $\theta$ curve.) We may assume that $Z_{2}^{\prime} \cap Z_{3}=Z_{2} \cap Z_{3}$. By the compactness of $Y, Z_{2}^{\prime}$ may be chosen close enough to $Z_{2}$ so that either $f\left(Z_{2}^{\prime}\right) \cap Z_{3}=Z_{2} \cap Z_{3}$ (Case (a)) or $Z_{2}^{\prime} \cap f\left(Z_{3}\right)=Z_{2} \cap Z_{3}$ (Case (b)), for all $f \in Y$. Let $Z_{2}^{\prime \prime}$ be the disk such that $Z_{2}^{\prime}$ $=Z_{2}+Z_{2}^{\prime \prime}$ and Int $\left(Z_{2}\right) \cap \operatorname{Int}\left(Z_{2}^{\prime \prime}\right)=\varnothing$. Let $\left\{R_{t}\right\}_{t \in I}$ be a continuous (in $t$ ) family of 
homeomorphisms of $Z$ into $Z$ such that $R_{0}=\mathrm{Id} ; R_{t}(p)=p$ if $p \notin Z_{2}^{\prime}$, for all $t \in I$; and $R_{1}\left(Z_{2}^{\prime}\right)=Z_{2}^{\prime \prime}$. Define $\lambda: Y \times I \rightarrow V A N$ by

$$
\begin{aligned}
\lambda(f, t)(x) & =x & & \text { if } x \notin R_{t}(Z), \\
& =R_{t} f R_{t}^{-1}(x) & & \text { if } x \in R_{t}(Z)
\end{aligned}
$$

for all $(f, t) \in Y \times I$. Then $\lambda$ is the required homotopy. $\lambda$ satisfies the last condition of the lemma if we choose $Z_{2}^{\prime}$ inside the neighborhood $N$.

The proof of Lemma 12 is complete.

We now return to the lemmas used in proving Theorem 3.

Proof of Lemma 7. (We assume the reader is familiar with the notation used in Step B of the proof of Theorem 3.) We want to show that the spaces $Q, K_{1}$, and the map $\pi_{1}: Q \rightarrow K_{1}$ satisfy the hypotheses of Theorem 6 . We know that $Q$ is complete. $K_{1}$ is finite dimensional since it is homeomorphic to $K$.

$\pi_{1}$ is onto. Fix $f \in K_{1}$. Let $H O L$ be the disk bounded by $B_{1}$ plus a subarc of either the "top" or "bottom" of Bd $\left(H_{1}\right)$. Let $p, q$ be points of $B_{1} \cap \operatorname{Int}\left(H_{1}\right)$ such that $B_{1} \cap f\left(l_{1}\right) \subset F-\{p, q\}$, where $F$ is the subarc of $B_{1}$ with endpoints $p, q$. It is clear that $f\left(l_{1} \cap \sum_{i=1}^{n} m_{i}\right)$ lies outside $H O L$, so $f\left(l_{1}\right) \cap H O L$ and $f\left(\sum m_{i}\right) \cap H O L$ are disjoint closed subsets of $H O L$. By [18, 16.2, p. 127] there are two spanning arcs $F_{1}, F_{2}$ of $H O L$ from $p$ to $q$ such that $F_{1} \cap F_{2}=\{p, q\}$, and the disk bounded by $F_{1}+F_{2}$ misses $f\left(l_{1}+\sum m_{i}\right)$ (and thus separates $f\left(l_{1}\right) \cap H O L$ and $f\left(\sum m_{i}\right) \cap H O L$ in $H O L)$. Let $F_{3}$ be a spanning arc of the closure of $H_{1}-H O L$, from $p$ to $q$, chosen close enough to $F$ so that the disk bounded by $F+F_{3}$ misses $\left|N S E P-B_{1}\right|+f\left(\sum m_{i}\right)$. Combining the disks bounded by $F+F_{3}, F+F_{2}, F_{1}+F_{2}$ with the arc $B_{1}$ gives us the homeomorphic image of $M O N$ which we require.

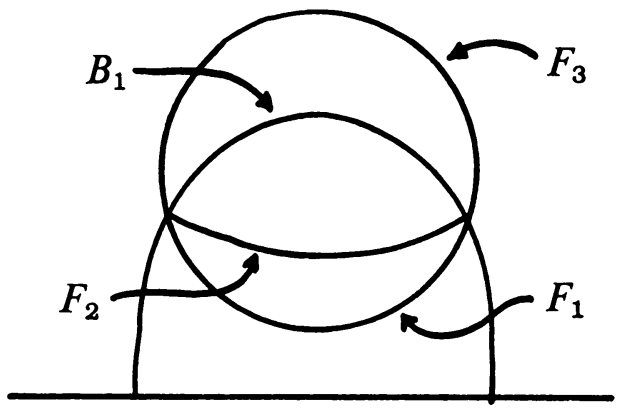

Diagram 4

$\pi_{1}$ is open. Suppose $\left(f, j_{0}\right) \in W$, and $W$ is open in $Q$. Then for sufficiently small $\varepsilon$, if $\operatorname{dist}(f, g)<\varepsilon$, then $\left(g, j_{0}\right) \in W$.

Each inverse set is homotopically trivial. Fix $f \in K_{1}$. Let $\alpha$ be a map of an $n$ sphere $S^{n}$ into $\pi_{1}^{-1}(f)$. Let

$$
X=\left\{j \in H\left(M O N, H_{1}\right):(f, j) \in \alpha\left(S^{n}\right)\right\} .
$$


It suffices to construct a map $\lambda: C(X) \rightarrow H\left(M O N, H_{1}\right)$ such that $\lambda(j, 0)=j$, all $j \in X$, and $\{f\} \times \lambda(C(X) \subset Q$.

Let $H O L$ be the disk defined above. We note that if $j \in X$, then $j\left(D_{1}\right) \subset H O L$, rather than $j\left(D_{3}\right) \subset H O L$ (by property (6) in the definition of $Q$ ). Since the compact set $\left|\left\{j\left(D_{3}\right): j \in X\right\}\right|$ is disjoint from $f\left(\sum m_{i}\right)+\left|N S E P-B_{1}\right|$ there is a spanning arc in the closure of $H_{1}-H O L$, joining the endpoints of $B_{1}$, such that the disk $O U T$ bounded by this arc and $B_{1}$ contains $\left|\left\{j\left(D_{3}\right): j \in X\right\}\right|$ but misses $f\left(\sum m_{i}\right)$ $+\left|N S E P-B_{1}\right|$ (by $[18,16.2$, p. 127]). Similarly there is a spanning arc in $H O L$ joining the endpoints of $B_{1}$ such that the disk $I N$ bounded by this arc and $B_{1}$ contains $\left|\left\{j\left(D_{1}+D_{2}\right): j \in X\right\}\right|$ but misses $f\left(\sum m_{i}\right)$. We shall shrink $X$ to a point so that if $i$ is in the image of $C(X)$ then $i(M O N) \subset I N+O U T$, thus $i\left(D_{3}\right) \cap\left|N S E P-B_{1}\right|$ $=\varnothing$, and $i(M O N)$ misses $f\left(\sum m_{i}\right)$.

We shrink $X$ in four stages.

Stage 1. Let $F$ be an open subarc of $B_{1}$ such that $f\left(l_{1}\right) \cap B_{1} \subset F$, and

$$
F \cap\left|\left\{j\left(M_{1}+M_{2}\right): j \in X\right\}\right|=\varnothing
$$

(recall that $M_{1}, M_{2}$ were the "stickers" attached to $D_{1}+D_{2}+D_{3}$ ). (We assume here that $f\left(l_{1}\right) \cap B_{1} \neq \varnothing$. If $f\left(l_{1}\right) \cap B_{1}=\varnothing$ we choose the points $b_{1}, b_{2}$, mentioned below, to be any two points in $B_{1} \cap \operatorname{Int}\left(H_{1}\right)$ and define isotopies $G_{t}^{p, q}$ similar to those defined below.) Let $b_{1}, b_{2}$ be fixed points of Int $\left(H_{1}\right)$ in different components of $B_{1}-F$. For all pairs of points $p, q$ of Int $\left(H_{1}\right), p, q$ in different components of $B_{1}-F$, we may construct a canonically defined isotopy $G_{t}^{p, q}$ of $I N+O U T$ onto itself which is fixed on $\mathrm{Bd}(I N+O U T)$, which takes $B_{1}$ onto itself, which moves the points $p, q$ onto the points $b_{1}, b_{2}$, and which is the identity outside a preassigned neighborhood of $B_{1}-F$. Using the isotopies $G_{t}^{p, q}$ we may obtain a homotopy $\lambda_{1}: X \times I \rightarrow H\left(M O N, H_{1}\right)$ such that for all $(j, t) \in X \times I$ we have $\lambda_{1}(j, 0)=j$, $\lambda_{1}(j, t)(M O N) \subset I N+O U T,\{f\} \times \lambda_{1}(X \times I) \subset Q$, and $\lambda_{1}(j, 1)\left(e_{i}\right)=b_{i}, i=1,2$ (recall that $e_{1}, e_{2}$ are the endpoints of the arc $\left.D_{2} \cap D_{3}\right)$. Let $X_{1}=\lambda_{1}(X \times\{1\})$.

Stage 2. Let $j_{1}$ be a fixed element of $X_{1}$. We want to get a homotopy $\lambda_{2}: X_{1} \times I \rightarrow H\left(M O N, H_{1}\right)$ such that for all $(j, t) \in X_{1} \times I$, we have $\lambda_{2}(j, 0)=j$, $\lambda_{2}(j, t)(M O N) \subset I N+O U T,\{f\} \times \lambda_{2}\left(X_{1} \times I\right) \subset Q$, and $\lambda_{2}(j, 1)\left|M_{3}=j_{1}\right| M_{3}$.

Since the compact set $\left|\left\{j\left(D_{1}\right): j \in X_{1}\right\}\right|$ is disjoint from $f\left(l_{1}\right)$ there is a spanning arc $C U T$ in $H O L$, from $b_{1}$ to $b_{2}$, such that $C U T$ separates $f\left(l_{1}\right)$ and $\left|\left\{j\left(D_{1}\right): j \in X_{1}\right\}\right|$ $-\left\{b_{1}+b_{2}\right\}$ in $H O L$. Let $H\left(B_{1}\right)$ denote the space of homeomorphisms of $B_{1}$ onto itself which are fixed on the endpoints of $B_{1}$ and on $b_{1}, b_{2}$. There is a map $\mu: H\left(B_{1}\right) \times I \rightarrow H\left(B_{1}\right)$, defined similarly to the Alexander map $A L$ of Lemma 1 , which contracts $H\left(B_{1}\right)$ to $\{\mathrm{Id}\} \subset H\left(B_{1}\right)$. Also, for each $(h, t) \in H\left(B_{1}\right) \times I$, we may extend $\mu(h, t)$ in a canonical fashion to a homeomorphism of $I N+O U T$ onto itself which is the identity on $\mathrm{Bd}(I N+O U T)+C U T$. Identify $\mu(h, t)$ and its extension. Then the homotopy $\lambda_{2}: X_{1} \times I \rightarrow H\left(M O N, H_{1}\right)$ is defined by

$$
\lambda_{2}(j, t)=\mu\left(j_{1} j^{-1} \mid B_{1}, 1-t\right) \circ j .
$$


Note that for all $(j, t) \in X_{1} \times I, \lambda_{2}(j, t)\left(D_{1}\right)$ misses $f\left(l_{1}\right)$, since $\mu\left(j_{1} j^{-1} \mid B_{1}, 1-t\right)=\mathrm{Id}$ on CUT. Let $X_{2}=\lambda_{2}\left(X_{1} \times\{1\}\right)$.

Stage 3. Let $F I X$ be the disk bounded by $C U T$ plus a subarc of $B_{1}$. We want a homotopy $\lambda_{3}: X_{2} \times I \rightarrow H\left(M O N, H_{1}\right)$ which satisfies the usual three conditions plus $\lambda_{3}(j, 1)=j_{1}$ on $M_{3}+j_{1}^{-1}(F I X)\left(j_{1}\right.$ is the fixed element mentioned in Stage 2). If we let $Z, Z_{1}, Z_{2}, Z_{3}, Y$ be represented by $I N, j_{1}\left(M_{3}+D_{1}+D_{2}\right), F I X, j_{1}\left(D_{1}\right)$, $\left\{j j_{1}^{-1}: j \in X_{2}\right\}$, respectively, then by Lemma $12(\mathrm{~b})$, there is a homotopy $\mu: X_{2} \times I \rightarrow H\left(Z_{1}, Z\right)$ such that $\mu(j, 0)=j j_{1}^{-1}$ and $\mu(j, 1)=\operatorname{Id}$ on $F I X$, all $j \in X_{2}$. Define $\lambda_{3}: X_{2} \times I \rightarrow H\left(M O N, H_{1}\right)$ by $\lambda_{3}(j, t)=j$ on $D_{3}, \lambda_{3}(j, t)=\mu(j, t) \circ j_{1}$ on $M_{3}+D_{1}+D_{2}$. Note that for all $(j, t) \in X_{2} \times I, \lambda_{3}(j, t)\left(D_{1}\right)$ misses $f\left(l_{1}\right)$, since, by the defining property of $V A N, \lambda_{3}(j, t)\left(D_{1}\right)$ misses the interior of $F I X$. Let $X_{3}$ $=\lambda_{3}\left(X_{2} \times\{1\}\right)$.

Stage 4. We complete the shrinking. If we let $Z, Z_{1}$ be represented by $O U T$, $j_{1}\left(D_{3}\right)$, resp., then by Lemma $11(\mathrm{a})$, there is a homotopy $\psi: X_{3} \times I \rightarrow H\left(Z_{1}, Z\right)$ such that $\psi(j, 0)=j j_{1}^{-1}$ and $\psi(j, 1)=\mathrm{Id}$, all $j \in X_{3}$. Define a homotopy $\lambda_{4}: X_{3} \times I \rightarrow H\left(M O N, H_{1}\right)$ by $\lambda_{4}(j, t)=j$ on $M_{3}+D_{1}+D_{2}, \lambda_{4}(j, t)=\psi(j, t) \circ j_{1}$ on $D_{3}$. Then for all $j \in X_{3}, \lambda_{4}(j, 1)=j_{1}$ except on $\left(D_{1}+D_{2}\right)-j_{1}^{-1}(F I X)$. Let $X_{3}$ $=\lambda_{4}\left(X_{3} \times\{1\}\right)$. If we let $Z, Z_{1}$ now be represented by the closures of $I N-F I X$ and $j_{1}\left(D_{1}+D_{2}\right)-F I X$, resp., then another application of Lemma 11(a) shrinks $X_{4}$ to the point $j_{1} \in X_{4}$. This completes the shrinking and demonstrates that $\pi_{1}^{-1}(f)$ is homotopically trivial.

An analogous, though easier (omits Stage 3), proof shows that $\pi_{1}^{-1}(f)$ is $\mathrm{LC}^{n}$ for all $n$ (see definition of $\mathrm{LC}^{n}$ in $\S 2$ ), and all $f \in K_{1}$.

$\left\{\pi_{1}^{-1}(f)\right\}_{f \in K_{1}}$ is equi-LC ${ }^{n}$ for all $n$. Fix $\left(f, j_{0}\right) \in Q$, an integer $n$, and $\varepsilon>0$. Let $W$ be an $\varepsilon / 2$ neighborhood of $\left(f, j_{0}\right)$ in $K_{1} \times H\left(M O N, H_{1}\right)$. Assume $\varepsilon$ is small enough so that if $(h, j) \in K_{1} \times H\left(M O N, H_{1}\right)$, and $j\left(M_{3}\right)=B_{1}$, and dist $\left((h, j),\left(f, j_{0}\right)\right)<\varepsilon$, then $(h, j) \in Q$. Since $\pi_{1}^{-1}(f)$ is $\mathrm{LC}^{n}$ we may choose a $\delta$-neighborhood $U^{\prime}$ of $\left(f, j_{0}\right), \delta<\varepsilon / 2$, such that any mapping of a $k$-sphere, $k \leqq n$, into $\pi_{1}^{-1}(f) \cap U^{\prime}$ is homotopic in $\pi_{1}^{-1}(f) \cap W$ to a constant. Let $U^{\prime \prime}$ be a $\delta / 2$ neighborhood of $\left(f, j_{0}\right)$. Let $\alpha$ be a map of a $k$-sphere $S^{k}$ into $\pi_{1}^{-1}(g) \cap U^{\prime \prime}$ for some $g \in K_{1}$. Define $\gamma: \pi_{1}^{-1}(g) \rightarrow \pi_{1}^{-1}(f)$ by $\gamma(g, j)=(f, j)$. Then $\gamma \alpha$ maps $S^{k}$ into $\pi_{1}^{-1}(f) \cap U^{\prime}$, so $\gamma \alpha$ has an extension $\bar{\alpha}: C\left(S^{k}\right) \rightarrow \pi_{1}^{-1}(f) \cap W$. But then $\gamma^{-1} \bar{\alpha}$ is the required map of $C\left(S^{k}\right)$ into $\pi_{1}^{-1}(g) \cap \varepsilon$-neighborhood of $\left(f, j_{0}\right)$.

Thus by Theorem $6, \pi_{1}$ has a right inverse.

The proof of Lemma 7 is complete.

The following lemma may be proved using the classical Schoenflies theorem and elementary plane topology (see [18]).

LEMMA 13. Let $A$ be a spanning arc of a disk $Z, \varepsilon$ a positive number, and $h a$ homeomorphism of $Z$ onto $Z$ which is fixed on $\mathrm{Bd}(Z)$. Then there is a positive number $\delta$ such that given any homeomorphism $j$ of $A$ onto a spanning arc of $Z$ having the same endpoints as $A$, with $\operatorname{dist}(j, h \mid A)<\delta$, there is a homeomorphism $g$ of $Z$ onto $Z$, fixed on $\mathrm{Bd}(Z)$, such that $g \mid A=j$, and dist $(g, h)<\varepsilon$. 
(Proof omitted.)

We use Lemma 13 in the

Proof of Lemmas 8 and 9. (We assume the reader is familiar with the notation used in Step C of the proof of Theorem 3.) The proofs of Lemmas 8 and 9 are almost identical. Therefore, we shall assume Lemma 8 has been proved and proceed with the proof of Lemma 9. The proof consists of verifying that the hypotheses of Theorem 6 hold for the spaces $L I F, K_{2} \times I$, and the map $\pi_{12}: L I F \rightarrow K_{2} \times I$. LIF is complete, since the set of all $(f, t, h)$ satisfying $\lambda(f, t)=h \mid A$ is closed, and all $(f, t, h)$ satisfying $h(\operatorname{MIS}(f)) \cap|S E P|=\varnothing$ is open in $K_{2} \times I \times H\left(H_{1}^{\prime}\right)$. (LIF is a $G_{\delta}$ in a complete metric space.) Since $K_{2}$ is the homeomorphic image of $K$, we see that $K_{2} \times I$ is finite dimensional. Before verifying the rest of the conditions we must stop and analyze the sets $\operatorname{MIS}(f), f \in K_{2}$ (if we were proving Lemma 8, we would analyze $\left.f\left(\sum m_{i}\right), f \in K_{2}\right)$. Fix $f \in K_{2}$. Since $\left(r_{1}(f)^{-1} \circ f\right)\left(l_{1}\right)=j_{0}\left(l_{1}\right)=A$, we see that for each vertical crosscut $m_{i}, 1 \leqq i \leqq n,\left(r_{1}(f)^{-1} \circ f\right)\left(m_{i}\right)$ intersects the arc $A$ in exactly one point (the point $j_{0}\left(p_{i}\right)$, which is the "left" endpoint of the arc $\left.A_{i}\right)$. Thus there is only one component of $\left(r_{1}(f)^{-1} \circ f\right)\left(m_{i}\right) \cap \operatorname{Int}\left(H_{1}^{\prime}\right)$ which intersects $A$. Denote this component (open arc) by $E_{i}$. Since $A$ separates the "top" and "bottom" of $H_{1}^{\prime}$, all other components of $\left(r_{1}(f)^{-1} \circ f\right)\left(m_{i}\right) \cap \operatorname{Int}\left(H_{1}^{\prime}\right)$ have either both endpoints in the "top" or both endpoints in the "bottom" of $H_{1}^{\prime}$. Let $P I$ denote the set $\left\{E_{1}, \ldots, E_{n}\right\}$. Since the set $|S E P|$ separates $f\left(m_{i}\right)$ and $f\left(m_{j}\right), i \neq j$, in $H_{1}^{\prime}$, and since MIS $(f) \cap|S E P|=\varnothing$, we see that $|S E P|$ separates $E_{i}$ and $E_{j}, i \neq j$. Thus each component of $H_{1}^{\prime}-|S E P|$ can contain at most one element of $P I$.

We now choose a finite collection $\left\{F_{\alpha}\right\}$ of spanning arcs of $H_{1}^{\prime}$ which will separate MIS $(f)-|P I|$ from $A+|S E P|$. Let $G_{\alpha}$ be one of the (finitely many) components of Int $\left(H_{1}^{\prime}\right)-(|S E P|+|P I|+A)$. Then $\mathrm{Bd}\left(G_{\alpha}\right)$ is a simple closed curve, $\mathrm{Bd}\left(G_{\alpha}\right)$ $\cap \mathrm{Bd}\left(H_{1}^{\prime}\right)$ is an arc, and the only limit points in $\mathrm{Bd}\left(G_{\alpha}\right)$ of $\mathrm{MIS}(f) \cap G_{\alpha}$ lie in the $\operatorname{arc} \mathrm{Bd}\left(G_{\alpha}\right) \cap \mathrm{Bd}\left(H_{1}^{\prime}\right)$. Let $F_{\alpha}^{\prime}$ be a subarc of Bd $\left(G_{\alpha}\right) \cap \mathrm{Bd}\left(H_{1}^{\prime}\right)$ which contains all limit points in $\operatorname{Bd}\left(G_{\alpha}\right)$ of MIS $(f) \cap G_{\alpha}$, and which misses $|S E P|$. Finally, choose $F_{\alpha}$ to be a spanning arc of $G_{\alpha}$, with the same endpoints as $F_{\alpha}^{\prime}$, such that

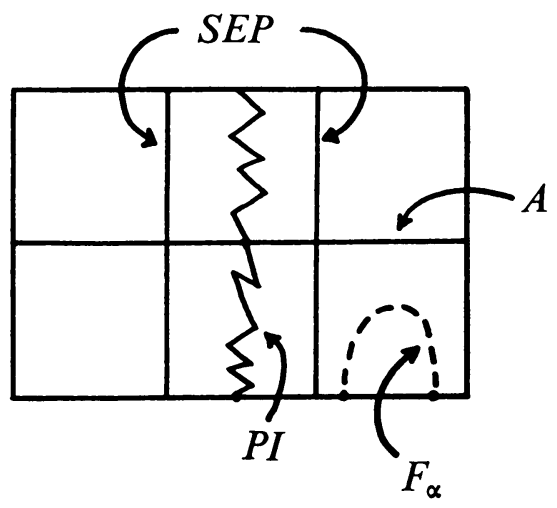

DIAGRAM 5 
$F_{\alpha} \cap \operatorname{MIS}(f) \cap G_{\alpha}=\varnothing$. This defines the (finite) collection $\left\{F_{\alpha}\right\}$. For future reference note that each $F_{\alpha}$ can be chosen to lie in a preassigned neighborhood of $F_{\alpha}^{\prime}+\left(G_{\alpha} \cap \operatorname{MIS}(f)\right.$ ). (If we were proving Lemma 8, we would replace MIS $(f)$ above by $f\left(\sum m_{i}\right), A$ by $f\left(l_{1}\right)$, and choose similar $F_{\alpha}$ 's.)

$\pi_{12}$ is onto. Fix $(f, t) \in K_{2} \times I$. Let $P I$ and $\left\{F_{\alpha}\right\}$ be defined as above for our given $f \in K_{2}$. We want to find $h \in H\left(H_{1}^{\prime}\right)$ so that $(f, t, h) \in L I F$. We start by defining $h$ on $A$ as equal to $\lambda(f, t)$. Now, if $\alpha$ is an arc of $S E P$, then $A_{i} \cap \alpha=\varnothing$ if and only if $\lambda(f, t)\left(A_{i}\right) \cap \alpha=\varnothing, 1 \leqq i \leqq n$ (by our choice of $\lambda$ ). Therefore, for each arc $E_{i} \in P I$, we may choose a corresponding spanning arc $E_{i}^{\prime \prime}$, with the same endpoints as $E_{i}$, such that $E_{i}^{\prime \prime} \cap|S E P|=\varnothing$, and $E_{i}^{\prime \prime} \cap h(A)=h\left(E_{i} \cap A\right)=h\left(j_{0}\left(p_{i}\right)\right)$. We may then extend $h$ to a homeomorphism of $\mathrm{Bd}\left(H_{1}^{\prime}\right)+A+\sum E_{i}$ onto $\mathrm{Bd}\left(H_{1}^{\prime}\right)+h(A)+\sum E_{i}^{\prime \prime}$ in the obvious way. Now, for each $\operatorname{arc} F_{\alpha}$ we pick a corresponding spanning arc $F_{\alpha}^{\prime \prime}$, with the same endpoints as $F_{\alpha}$, such that $F_{\alpha}^{\prime \prime} \cap \operatorname{Int}\left(H_{1}^{\prime}\right)$ misses $|S E P|+h(A)$ $+\sum E_{i}^{\prime \prime}$. Extend $h$ in the obvious way to a homeomorphism of $\mathrm{Bd}\left(H_{1}^{\prime}\right)+A+\sum E_{i}$ $+\sum F_{\alpha}$ onto $\mathrm{Bd}\left(H_{1}^{\prime}\right)+h(A)+\sum E_{i}^{\prime \prime}+\sum F_{\alpha}^{\prime \prime}$. Complete the extension by finitely many applications of the Schoenflies theorem. Now $(f, t, h) \in L I F$, since $h\left(\sum E_{i}\right) \cap|S E P|=\varnothing$, and the disks bounded by the $F_{\alpha}^{\prime \prime}$ 's plus the appropriate subarcs of the "top" and "bottom" of $H_{1}^{\prime}$ contain $h(\mathrm{MIS}(f)-|P I|)$, and do not intersect $|S E P|$.

Each inverse set is homotopically trivial. Fix $(f, t) \in K_{2} \times I$. Let $\alpha$ be a map of an $n$-sphere $S^{n}$ into $\pi_{12}^{-1}(f, t)$. Let $X=\left\{h \in H\left(H_{1}^{\prime}\right):(f, t, h) \in \alpha\left(S^{\eta}\right)\right\}$. It suffices to construct a map $\mu: C(X) \rightarrow H\left(H_{1}^{\prime}\right)$ such that $\mu(h, 0)=h$ for all $h \in X$, and $\{(f, t)\} \times \mu(C(X)) \subset L I F$.

Fix an element $h_{1} \in H\left(H_{1}^{\prime}\right)$. Suppose we can obtain a homotopy $\psi: X \times I \rightarrow H\left(H_{1}^{\prime}\right)$ such that for all $(h, t) \in X \times I$, (a) $\psi(h, 0)=h_{1}^{-1} h, \psi(h, 1)=\mathrm{Id}$, (b) $\psi(h, t) \mid A=\mathrm{Id}$, and (c) $\psi(h, t)(\operatorname{MIS}(f)) \cap h_{1}^{-1}(|S E P|)=\varnothing$. Then the required shrinking $\mu$ will be defined by $\mu(h, t)=h_{1} \circ \psi(h, t)$, all $(h, t) \in X \times I$.

It remains to construct $\psi$. Let $P I,\left\{F_{\alpha}\right\}$ be defined as above for our given $f \in K_{2}$. For each $F_{\alpha}$, let $F_{\alpha}^{\prime}$ be the subarc of either "top" or "bottom" of Bd $\left(H_{1}^{\prime}\right)$ cut off by the endpoints of $F_{\alpha}$. Note that $F_{\alpha}^{\prime} \cap|S E P|=\varnothing$. We may choose $F_{\alpha}$ so that every point of $F_{\alpha}$ is within a preassigned distance of $F_{\alpha}^{\prime}+\operatorname{MIS}(f)$. Therefore, since $\left\{h_{1}^{-1} h: h \in X\right\}$ is a compact set of homeomorphisms, and the compact set $\left|\left\{h_{1}^{-1} h(\mathrm{MIS}(f)): h \in X\right\}\right|$ is disjoint from $h_{1}^{-1}(|S E P|)$, we may assume that the set $\left|\left\{h_{1}^{-1} h\left(F_{\alpha}\right): h \in X\right\}\right|$ is disjoint from $h_{1}^{-1}(|S E P|)$, for each $F_{\alpha}$. Hence, if $J_{\alpha}$ is the disk bounded by $F_{\alpha}+F_{\alpha}^{\prime}$, we have that $\left|\left\{h_{1}^{-1} h\left(J_{\alpha}\right): h \in X\right\}\right|$ misses $h_{1}^{-1}(|S E P|)$, for each $J_{\alpha}$. Fix a $J_{\alpha}$. Now if we let $Z, Z_{1}, Z_{2}, Z_{3}, Y$ be represented by $H_{1}^{\prime}, H_{1}^{\prime}, J_{\alpha}$, $h_{1}^{-1}(|S E P|),\left\{h_{1}^{-1} h: h \in X\right\}$, resp., then by Lemma $12(\mathrm{a})$, there is a homotopy $\psi_{1}: X \times I \rightarrow H\left(H_{1}^{\prime}\right)$ such that for all $h \in X, \psi_{1}(h, 0)=h_{1}^{-1} h, \psi_{1}(h, 1) \mid J_{\alpha}=\mathrm{Id}$, and $\psi_{1}$ has the other two required properties. Let $X_{1}=\psi_{1}(X \times\{1\})$. We may assume by induction that if $h \in X_{1}$, then $h \mid J_{\alpha}=$ Id for all $J_{\alpha}$. Let $H_{1}^{\prime \prime}$ be the closure of $H_{1}^{\prime}-\sum J_{\alpha}$. Then $|P I|+A+h_{1}^{-1}(|S E P|) \subset H_{1}^{\prime \prime}$, and $h=\operatorname{Id}$ on $\operatorname{Bd}\left(H_{1}^{\prime \prime}\right)$, all $h \in X_{1}$. Now if we let $Z, A,\left\{\alpha_{1}, \ldots, \alpha_{n-1}\right\},\left\{\gamma_{1}, \ldots, \gamma_{m}\right\}$ be represented by $H_{1}^{\prime \prime}, A, P I,\left\{h_{1}^{-1}(\beta): \beta \in S E P\right\}$, 
resp., then by Lemma 11(c) the set $X_{1}$ may be shrunk to the Id in the required way. Thus $\pi_{12}^{-1}(f, t)$ is homotopically trivial. (This step for Lemma 8 is somewhat easier; $h_{1}^{-1}(|S E P|)$ may be replaced by $|S E P|$.)

A proof using only the Alexander map $A L$ of Lemma 1 shows that $\pi_{12}^{-1}(f, t)$ is $\mathrm{LC}^{n}$ for all $n$, all $(f, t) \in K_{2} \times I$.

We prove that $\pi_{12}$ is open and equi-LC ${ }^{n}$, for all $n$, at the same time. Fix $\left(f, t_{1}, h_{1}\right) \in L I F$, an integer $n$, and a positive number $\varepsilon$. Assume $\varepsilon$ is small enough so that if $(g, t, h) \in K_{2} \times I \times H\left(H_{1}^{\prime}\right)$, and dist $\left(\left(f, t_{1}, h_{1}\right),(g, t, h)\right)<\varepsilon$, then $h(\operatorname{MIS}(g))$ $\cap|S E P|=\varnothing$. Let $W$ be an $\varepsilon / 4$ neighborhood of $\left(f, t_{1}, h_{1}\right)$. Since $\pi_{12}^{-1}\left(f, t_{1}\right)$ is LC ${ }^{n}$, we may choose a $\delta$ neighborhood $U^{\prime}$ of $\left(f, t_{1}, h_{1}\right), \delta<\varepsilon / 4$, so that any map of a $k$-sphere, $k \leqq n$, into $U^{\prime} \cap \pi_{12}^{-1}\left(f, t_{1}\right)$ is homotopic in $W \cap \pi_{12}^{-1}\left(f, t_{1}\right)$ to a constant. By Lemma 13 and the continuity of $\lambda$, there is a $\delta_{1}$ neighborhood $U^{\prime \prime}$ of $\left(f, t_{1}, h_{1}\right)$, $\delta_{1}<\delta / 4$, such that if $(g, t) \in K_{2} \times I$, and dist $\left((g, t),\left(f, t_{1}\right)\right)<\delta_{1}$, then there is a map $h_{2} \in H\left(H_{1}^{\prime}\right)$ such that $h_{2} \mid A=\lambda(g, t)$, and dist $\left(h_{1}, h_{2}\right)<\delta / 2$. (Thus $\left(g, t, h_{2}\right)$ $\in L I F$, and $\left(g, t, h_{2}\right) \in U^{\prime}$, hence $\pi_{12}$ is an open map.) Now let $\alpha$ be a map of a $k$-sphere $S^{k}$ into $\pi_{12}^{-1}(g, t) \cap U^{\prime \prime}$ for some $(g, t) \in K_{2} \times I$. Choose $h_{2}$ for $(g, t)$ as above. Define a map $\gamma: \pi_{12}^{-1}(g, t) \rightarrow \pi_{12}^{-1}\left(f, t_{1}\right)$ by $\gamma(g, t, h)=\left(f, t_{1}, h_{1} h_{2}^{-1} h\right)$. Since dist $\left(h_{1} h_{2}^{-1}(x), x\right)<\delta / 2$ for all $x \in H_{1}^{\prime}$, we see that $\gamma \alpha$ maps $S^{k}$ into $U^{\prime} \cap \pi_{12}^{-1}\left(f, t_{1}\right)$. Hence there is an extension $\bar{\alpha}$ mapping $C\left(S^{k}\right)$ into $W \cap \pi_{12}^{-1}\left(f, t_{1}\right)$. But then $\gamma^{-1} \bar{\alpha}$ is the required mapping into $\pi_{12}^{-1}(g, t)$ and the $\varepsilon$ neighborhood of $\left(f, t_{1}, h_{1}\right)$.

Hence, by Theorem 6 , the map $\pi_{12}$ has a right inverse.

The proof of Lemma 9 is complete.

Proof of Lemma 10. (We assume the reader is familiar with the notation used in Step $\mathrm{D}$ of the proof of Theorem 3.) That the spaces $V E R, K_{3}$, and the map $\pi_{1}$ satisfy the hypotheses of Theorem 6 is easily verified. $K_{3}$, being the homeomorphic image of $K$, is finite dimensional. $V E R$, being a closed subset of the complete metric space $K_{3} \times H\left(D_{1}\right)$, is a complete metric space. $\pi_{1}$ is onto by the Schoenflies theorem. For each $f \in K_{3}, \pi_{1}^{-1}(f)$ is $\mathrm{LC}^{n}$, all $n$, and homotopically trivial by application of the Alexander map $A L$ of Lemma 1. $\pi_{1}$ is open, and $\left\{\pi_{1}^{-1}(f)\right\}_{f \in K_{3}}$ is equi-LC ${ }^{n}$, all $n$, by Lemma 13 and the fact that $\pi_{1}^{-1}(f)$ is $\mathrm{LC}^{n}$, all $f \in K_{3}$. This completes the proof.

Lemma 14. Suppose $K$ is a compact subset of $H(D), \varepsilon$ a positive number, and $\psi_{1}: C(K) \rightarrow H(D)$ a map such that $\psi_{1}(f, 0)=f$, all $f \in K$, and for each $t \in[0,1)$, $\psi_{1} \mid K \times\{t\}$ is a homeomorphism. Then there is an embedding $\psi: C(K) \rightarrow H(D)$ such that $\psi(f, 0)=f$, all $f \in K$, and dist $\left(\psi_{1}(f, t), \psi(f, t)\right)<\varepsilon$ for all $(f, t) \in C(K)$.

Proof. Let $X=\psi_{1}(C(K)) . X$ is compact, so by Ascoli's theorem [19, Chapter 4, $\S 25]$ there is a positive number $\delta, \delta<\varepsilon / 2$, so that if $x, y \in D$ and dist $(x, y)<\delta$, then $\operatorname{dist}(f(x), f(y))<\varepsilon / 2$ for all $f \in X$. We think of the disk $D$ as being the set $\left\{(x, y) \in R^{2}: 0 \leqq x \leqq 1,-1 \leqq y \leqq 1\right\}$, and denote the open arc $\{(x, 0): 0<x<\delta / 3\}$ by $A$. Choose a sequence $\left\{p_{n}\right\}_{n=1}^{\infty}$ of points of $A$ such that $\pi_{1}\left(p_{1}\right)>\pi_{1}\left(p_{2}\right)>\cdots$, and $\left\{\pi_{1}\left(p_{n}\right)\right\} \rightarrow 0\left(\pi_{1}: D \rightarrow I\right.$ is projection on first coordinate). Let $F \in H(D)$ be a 
homeomorphism such that dist $(F, \mathrm{Id})<\delta / 3$, and for each integer $n, 0<\pi_{1}\left(F\left(p_{n}\right)\right)$ $<\inf \left\{\pi_{1}\left(f^{-1}\left(p_{n}\right)\right): f \in X\right\}$. (Define $F$ in an order preserving manner from $\left\{p_{n}\right\}$ into $A$; extend linearly to a homeomorphism of $A$ onto itself; extend to $D$ by "coning".) Then for all $f \in X, f \circ F$ is not the identity in any neighborhood of the left side of $\mathrm{Bd}(D)$.

Let $S: R^{1} \rightarrow R^{1}$ be the homeomorphism defined by $S(x)=x$ if $\delta / 3 \leqq x, S(x)$ $=2 x-\delta / 3$ if $\delta / 6 \leqq x<\delta / 3, S(x)=x-\delta / 6$ if $x<\delta / 6$. Let $S_{t}: R^{1} \rightarrow R^{1}$ be the isotopy defined by $S_{t}(x)=t S(x)+(1-t) x$, all $t \in I$. Finally, let $R_{t}: R^{2} \rightarrow R^{2}$ be the isotopy defined by $R_{t}(x, y)=\left(S_{t}(x), y\right)$, all $t \in I$. Note that dist $\left(R_{t}, \mathrm{Id}\right)<\delta / 3$, all $t \in I$.

The required embedding $\psi: C(K) \rightarrow H(D)$ is given by

$$
\psi(f, t)=R_{t}^{-1} \circ \psi_{1}(f, t) \circ F \circ R_{t} \circ F^{-1}, \quad \text { all }(f, t) \in C(K) .
$$

(We extend $\psi_{1}(f, t) \circ F$ outside $D$ by the identity.) It is easily checked that $\operatorname{dist}\left(\psi_{1}(f, t), \psi(f, t)\right)<\varepsilon$, all $(f, t) \in C(K)$. If $f, g \in K, f \neq g, t \in[0,1)$, then $\psi_{1}(f, t)$ $\neq \psi_{1}(g, t)$, so $\psi(f, t) \neq \psi(g, t)$. Suppose now $t, s \in I, f, g \in K$, and $s<t$. Let $l$ be the left side of Bd $(D)$; let $f_{1}=R_{s}^{-1} \circ \psi_{1}(f, s) \circ F \circ R_{s}$; and let $g_{1}=R_{t}^{-1} \circ \psi_{1}(g, t) \circ F \circ R_{t}$. Then $f_{1} \neq \mathrm{Id}$ in any neighborhood of $R_{s}^{-1}(l)$, but $g_{1}=$ Id in some neighborhood of $R_{s}^{-1}(l)$. Hence $f_{1} \neq g_{1}$ hence $\psi(f, s) \neq \psi(g, t)$. Hence $\psi$ is an embedding and the proof of Lemma 14 is complete.

\section{A corollary. An interesting consequence of Theorem 4 is}

Corollary 15. Suppose $K_{1}$ and $K_{2}$ are compact subsets of $H(D)$, and $\alpha$ is a homeomorphism of $K_{1}$ onto $K_{2}$. Then a may be extended to a homeomorphism of $H(D)$ onto itself.

We sketch the proof. Let $l_{2}$ be separable Hilbert space. By [10], $H(D)$ is homeomorphic to $l_{2} \times l_{2} \times H(D)$. Let $p$ be a fixed point of $l_{2} \times H(D)$. For each $i, i=1,2$, let $f_{i}$ be a homeomorphism of $l_{2} \times l_{2} \times H(D)$ onto itself such that $f_{i}\left(K_{i}\right) \subset\{0\} \times l_{2}$ $\times H(D)$. The existence of $f_{i}$ follows from [2, Corollary 10.2], since $\pi_{12}\left(K_{i}\right)$ is a compact subset of $l_{2} \times l_{2}\left(\pi_{12}\right.$ is projection on first two coordinates). For each $i$, $i=1,2$, there is an embedding $g_{i}: f_{i}\left(K_{i}\right) \rightarrow l_{2} \times\{p\}$ [14, Theorem 2-46]. Since $l_{2}$ is an AR, $g_{i}$ may be extended to a map of $\{0\} \times l_{2} \times H(D)$ into $l_{2} \times\{p\}$. By Theorem 4 of this paper $g_{i}^{-1}: g_{i} f_{i}\left(K_{i}\right) \rightarrow\{0\} \times l_{2} \times H(D)$ may be extended to a map of $l_{2} \times\{p\}$ into $\{0\} \times l_{2} \times H(D)$. But then, by [5, Lemma 1, Theorem 1], $g_{i}$ may be extended to a homeomorphism of $l_{2} \times l_{2} \times H(D)$ onto itself. Identify $g_{i}$ and its extension. By [2, Corollary 10.3], there is a homeomorphism $h$ of $l_{2} \times\{p\}$ onto itself such that $h \mid g_{1} f_{1}\left(K_{1}\right)=g_{2} f_{2} \alpha f_{1}^{-1} g_{1}^{-1}$. Extend $h$ to a homeomorphism of $l_{2} \times l_{2} \times H(D)$ onto itself, and identify $h$ with its extension. Then the required homeomorphism of $l_{2} \times l_{2} \times H(D)$ onto itself is $f_{2}^{-1} g_{2}^{-1} h g_{1} f_{1}$.

\section{BIBLIOGRAPHY}

1. J. W. Alexander, On the deformation of an n-cell, Proc. Nat. Acad. Sci. U.S.A. 9 (1923), 406-407. 
2. R. D. Anderson, On topological infinite deficiency, Michigan Math. J. 14 (1967), 365-383. MR 35 \#4893.

3. - Spaces of homeomorphisms of finite graphs (to appear).

4. R. D. Anderson and R. H. Bing, A complete elementary proof that hilbert space is homeomorphic to the countable infinite product of lines, Bull. Amer. Math. Soc. 74 (1968), 771-792. MR 37 \#5847.

5. M. Brown, Pushing graphs around, Conference on the Topology of Manifolds (Michigan State Univ., E. Lansing, Mich., 1967), Prindle, Weber \& Schmidt, Boston, Mass., 1968, pp. 19-22. MR 38 \#2785.

6. K. Borsuk, Über eine Klasse von lokal zusammenhangenden Raumen, Fund. Math. 19 (1932), 220-240.

7. C. H. Dowker, Mapping theorems for non-compact spaces, Amer. J. Math. 69 (1947), 200-242. MR 8, 594.

8. E. Dyer and M.-E. Hamstrom, Regular mappings and the space of homeomorphisms on a 2-manifold, Duke Math. J. 25 (1958), 521-531. MR 20 \#2695.

9. R. D. Edwards and R. C. Kirby, Deformations of spaces of imbeddings, Ann. of Math. (2) 93 (1971), 63-88.

10. R. Geoghegan, On spaces of homeomorphisms, embeddings and functions (to appear).

11. M.-E. Hamstrom, Regular mappings and the space of homeomorphisms on a 3-manifold, Mem. Amer. Math. Soc. No. 40 (1961). MR 27 \#2970.

12. - Some global properties of the space of homeomorphisms of a disc with holes, Duke Math. J. 29 (1962), 657-662. MR 26 \#745.

13. O. Hanner, Some theorems on absolute neighborhood retracts, Ark. Mat. 1 (1951), 389-408. MR 13, 266.

14. J. Hocking and G. Young, Topology, Addison-Wesley, Reading, Mass., 1961. MR 23 \#A2857.

15. W. Hurewicz and H. Wallman, Dimension theory, Princeton Math. Series, vol. 4, Princeton Univ. Press, Princeton, N. J., 1941. MR 3, 312.

16. W. Mason, The space $H(M)$ of homeomorphisms of a compact manifold onto itself is homeomorphic to $H(M)$ minus any o-compact set, Amer. J. Math. 92 (1970), 541-551.

17. E. Michael, Continuous selections. II, Ann. of Math. (2) 64 (1956), 562-580. MR 18, 325.

18. M. H. A. Newman, Elements of the topology of plane sets of points, 2nd ed., Cambridge Univ. Press, New York, 1961. MR 24 \#A2374.

19. G. Simmons, Introduction to topology and modern analysis, McGraw-Hill, New York, 1963. MR 26 \#4145.

20. A. H. Stone, Paracompactness and product spaces, Bull. Amer. Math. Soc. 54 (1948), 977-982. MR 10, 204.

21. G. T. Whyburn, Analytic topology, Amer. Math. Soc. Colloq. Publ., vol. 28, Amer. Math. Soc., Providence, R. I., 1942. MR 4, 86.

22. Problems on infinite-dimensional spaces and manifolds, Louisiana State University, Baton Rouge, La., 1969 (mimeographed).

Rutgers, The State University of New Jersey, New Brunswick, New Jersey 08903 